\title{
Knee Laxity or Loss of Knee Range of Motion after PCL Reconstruction: A Systematic Review and Meta-Analysis
}

\author{
1,2Sholahuddin Rhatomy ${ }^{*},{ }^{3,4}$ Dwikora Novembri Utomo, ${ }^{3,4}$ Heri Suroto, ${ }^{3,4}$ Ferdiansyah \\ Mahyudin \\ ${ }^{1}$ Department of Orthopedics and Traumatology, Dr. Soeradji Tirtonegoro General Hospital, Klaten, \\ Indonesia. ${ }^{2}$ Faculty of Medicine, Public Health, and Nursing, Universitas Gadjah Mada, Yogyakarta, \\ Indonesia. ${ }^{3}$ Department of Orthopaedic and Traumatology, Dr. Soetomo General Hospital, Surabaya, \\ Indonesia. ${ }^{4}$ Faculty of Medicine, Universitas Airlangga, Indonesia.
}

Submitted 16 August 2020; Accepted in final form 26 October 2020.

\begin{abstract}
Background. PCL reconstruction is a successful method for enhancing the patient's quality of life but posterior knee laxity and knee stiffness have still occurred surgery. There is no study to evaluate knee laxity or loss of knee range of motion after surgery. Objectives. To assess the outcomes after PCL reconstruction, we: 1) evaluated the range of motion of the knee, 2) evaluated posterior knee laxity, and 3) determined the factors that influence laxity or the loss of range of motion after surgery. Methods. Articles that met the following criteria were enrolled in this review: 1) articles on peer-reviewed level 1 to 4 studies; 2) articles published in English; 3) articles on PCL reconstruction studies; 4) articles on isolated PCL rupture; 5) articles that describe laxity after surgery and 6) articles that describe the degree of range of motion after surgery. Results. Involving a total of 1711 patients. There was a loss of extension and flexion after PCL reconstruction (9.15\% and $28.9 \%$, respectively). Knee laxity was still observed at the final examination in the posterior drawer test, KT 1000/2000 test, and Telos radiographic view (64.8\%, 42.8\%, and 47.9\%, respectively). In the subgroup analysis, there was no significant difference in laxity between allograft group vs autograft group using the KT 1000/2000 measurement (mean difference $[\mathrm{MD}]=-0.42,95 \%$ confidence interval $[-1.41,0.56], \mathrm{p}=0.40$ ), Single Bundle vs Double Bundle (DB) using the KT 1000/2000 measurement (MD $=-0.003,95 \%$ CI [-1.35, 1.29], $p$ $<0.00001$ ), and transtibial vs tibial inlay using the Telos radiograph measurement (MD $=0.03,95 \%$ CI $[-0.33,0.39]$, $\mathrm{p}=0.88)$, but $\mathrm{DB}$ significantly improved knee stability using the Telos radiographic measurement $(\mathrm{MD}=0.69,95 \%$ CI $[0.29,1.09], \mathrm{p}=0.00008)$. Conclusion. This study demonstrates that the loss of range of motion or laxity is still a problem after PCL reconstruction.
\end{abstract}

\section{KEYWORDS: Range of Motion, Laxity, Posterior Cruciate Ligament, PCL Reconstruction.}

\section{INTRODUCTION}

Only a few studies have investigated the outcome after posterior cruciate ligament (PCL) reconstruction, and the outcome of the results after surgery of these studies vary and need further depth research. Recent studies revealed that PCL reconstruction is a successful method for enhancing the patient quality of life and that it has a significant impact on patients' activity of daily living and back to the normal pre-injury activity, because can stabilize knee joint (1). However, in daily practice, we still observed and founded posterior knee laxity or knee stiffness after PCL reconstruction.

Posterior knee laxity or knee stiffness still always a problem after surgery and a challenge

*. Corresponding Author:

Sholahuddin Rhatomy, MD

E-mail: doktergustomrhatomy@yahoo.com 
for doctors and physiotherapists to prevent and manage it. There are many systematic reviews and meta-analyses on PCL reconstruction that have been reported before (1-8); however, none focused on laxity or stiffness after PCL reconstruction. This study aims to produce a systematic review and metaanalysis about laxity or stiffness of the knee after PCL reconstruction based on published literature.

\section{MATERIALS AND METHODS}

Review of Protocol. Our review question was "What is the incidence rate of posterior knee laxity or loss of range of motion of the knee after PCL reconstruction and what factors influence it?"

Outcomes Measure. To assess the outcomes after PCL reconstruction, we: 1) evaluated the range of motion of the knee, 2) evaluated posterior knee laxity, and 3) determined the factors that influence laxity or the loss of range of motion after surgery.

Literature Search and Study Selection. In May 2020, we carried out a literature search using Cochrane Library, PubMed (Medline), Web of Science, and Scopus to identify all the studies published in English that describe the outcomes after PCL reconstruction. All studies were reported based on the Preferred Reporting Items for Systematic Reviews and Meta-Analyses (PRISMA) statement (9).

The keywords used for the search included "laxity," "stiffness," "range of motion," "PCL," "Posterior Cruciate Ligament," "PCL Reconstruction," and "outcome" alone and in various combinations using the Boolean operator "AND" or "OR."

Eligibility Criteria. Inclusion criteria were: 1) articles on peer-reviewed level studies; 2) articles published in English; 3) articles on PCL reconstruction studies; 4) articles on isolated PCL rupture; 5) articles that describe laxity after surgery using the posterior drawer test, KT1000/KT-2000 test, and radiographic stress (Telos) view; and 6) articles that describe the degree of range of motion after surgery. Articles that met these inclusion criteria were enrolled in this systematic review.

Non-English articles, articles on multiple ligament reconstruction, articles on studies that involved PCL reconstruction combined with other techniques, duplicate articles, literature reviews, articles on studies that involve in vitro, animals, until the cadaveric investigation, biomechanical study, letters to editors, instructional courses, and technical notes were excluded. We also excluded articles with incomplete information on diagnosis, imaging, arthroscopic or surgical assessment of the associated lesions, clinical examination, follow-up duration, clinical postoperative outcomes, and no statistical analysis.

Data Extraction. To avoided bias, the following data were identified and recorded independently by all of the investigators: study design, types of graft, types of surgical technique, outcome after surgery, degree of knee laxity, range of motion, interventions, comparisons, duration of follow-up, main outcomes of studies, and complications.

Methodological Quality Assessment and Risk of Bias. The methodological quality of the included studies was assessed using the Preferred Reporting Items for Systematic Reviews and Meta-Analyses (PRISMA) statement (9). Two authors (D.N.U and S.R.) independently performed all the assessments.

Coleman Methodology Score (CMS) was used to quantify the quality of the article. The article's methodology was assessed by CMS with a total score ranging from 0 to 100 . The higher the CMS score of the article, the more valid its article because it spared from biases and confounding factors (10).

To avoid bias on the included and excluded articles were reviewed and re-assessed by all authors. If there was any disagreement between each author, the problem was solved by D.N.U. as a senior investigator.

Data Synthesis. We used RevMan 5 software (Version 5.3, the Cochrane Collaboration) and Stata 12.0 software for meta-analysis statistical analyses. The following tests were performed: the posterior drawer displacement test, KT 1000/2000 test, and the radiographic stress (Telos) view. The 95\% confidence interval (CI) and Mean Difference (MD) were counted for continuous data. The Odds Ratio (OR) and 95\% CI were calculated for dichotomous data. An alpha level of $<0.05$ was considered statistically significant.

The heterogeneity among the included studies was tested using the I-square tests and Chi-Square tests. The chi-square test was performed to quantify heterogeneity significance. The I-square test was performed to quantify the estimation of variability in the effect that occurred because of its heterogeneity. The result interpretation of the Isquare test was quantified based on the Cochrane Handbook of Systemic Reviews. The result has its interpretation (0-40\%, might not be important; 40$60 \%$, may represent moderate heterogeneity; 60 $90 \%$, may represent substantial heterogeneity; $90-$ 
$100 \%$, considerable heterogeneity). When there was no significant heterogeneity was present $\left(\mathrm{I}^{2}<\right.$ $50 \%, \mathrm{P}>0.1)$, the fixed-effect model was used. If the result was significant heterogeneity, we were used a random effect model. Sensitivity analyses were conducted by individually removing each study to assess the heterogeneity and robustness of the pooled results. Datasets that caused significant changes in the pooled results were further analyzed to assess the cause of the changes. Subsequently, the results were evaluated for stability and laxity. If the heterogeneity was significantly large for analysis, descriptive analyses were presented. Subgroup analyses were performed on the laxity outcome at different comparisons.

\section{RESULTS}

Study Selection. A total of 2041 articles were obtained from the database literature search and 1207 articles were excluded based on the title or due to duplication. A total of 834 articles were eligible for further screening. Seven-hundred-and-twenty-six articles were excluded because they did not match the inclusion criteria resulting in a total of 108 articles. Sixty-one articles were excluded after the full-text screening was performed. We excluded these articles due to: the range of motion or laxity after surgery was not clearly stated $(n=49)$, they were either technical notes, short communications, or reviews $(n=$ $6)$, they were cadaveric, laboratory, or biomechanical studies $(n=3)$, or they were nonoperative studies $(n=3)$. Thus, 47 full articles were included in this systematic qualitative review. The flow chart of the article enrolling was provided in Figure 1.

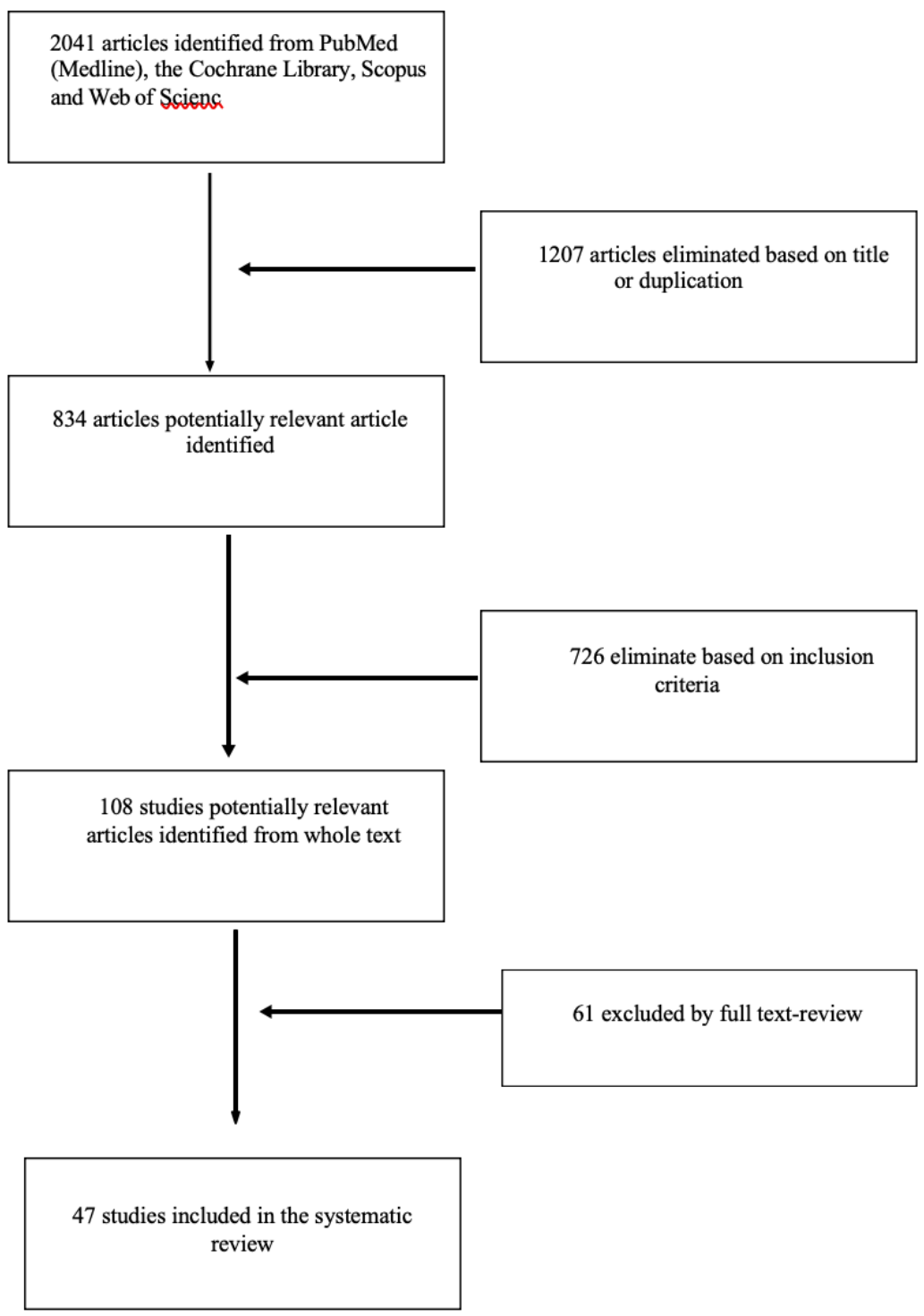

Figure 1. Flow Chart of Study Process Selection 
Demographics and Characteristics of Selected Studies. Twenty-three studies $(48.9 \%)$ were retrospective, 14 were prospective studies, six were case series, four were randomized control trials, and one was a non-randomized control trial. This systematic review included 1711 patients (1713 knees). There were 1293 male $(75.5 \%)$ and 385 female (22.5\%) patients. Two studies did not describe the gender distribution (33 patients). The average age at the time of reconstruction was 30.4 years (range, 16-64 years). The average follow-up duration was 44.85 months (range, 12-148 months) (Table 1).

In all studies, the indication for surgery was the failure of conservative treatment or symptomatic PCL rupture with a minimum 2 positive $(2+)$ on posterior drawer test.

Table 1. Summary of Characteristics of the Included Studi

\begin{tabular}{|c|c|c|c|c|}
\hline No & Author and Year & Study Type & Sample Size & Follow-Up Period \\
\hline 1 & P. P. Mariani et al,1997 (11) & $\begin{array}{l}\text { Retrospective } \\
\text { Study }\end{array}$ & 24 & $\begin{array}{c}26.5 \text { months (range } 24-53 \\
\text { months) }\end{array}$ \\
\hline 2 & $\begin{array}{c}\text { Sung-Jae Kim et al,2000 } \\
\text { (12) }\end{array}$ & Prospective Study & $\begin{array}{l}55 \text { (Two incision group: 10; One } \\
\text { incision group:45) }\end{array}$ & $\begin{array}{c}45 \text { months in Group I and } 36 \\
\text { months in group II }\end{array}$ \\
\hline 3 & John Nyland et al,2002 (13) & $\begin{array}{l}\text { Retrospective } \\
\text { Study }\end{array}$ & 19 & 2 years \\
\hline 4 & $\begin{array}{c}\text { Chih-Hwa Chen et al,2002 } \\
\text { (14) }\end{array}$ & Prospective Study & 27 & 2 years \\
\hline 5 & $\begin{array}{c}\text { Chih-Hwa Chen et al,2002 } \\
\text { (15) }\end{array}$ & Case Series Study & $\begin{array}{l}49 \text { ( } 22 \text { quadriceps tendon group } \\
\text { and } 27 \text { hamstring tendon group) }\end{array}$ & 2 years \\
\hline 6 & $\begin{array}{c}\text { Ching-Jen Wang et al,2003 } \\
(16)\end{array}$ & $\begin{array}{l}\text { Retrospective } \\
\text { Study }\end{array}$ & 30 & 40 (range: $24-108$ ) months \\
\hline 7 & $\begin{array}{c}\text { Yasumitsu Ohkoshi et } \\
\text { al,2003 (17) }\end{array}$ & $\begin{array}{l}\text { Nonrandomized } \\
\text { Control Study }\end{array}$ & $\begin{array}{l}\text { 51(The 2-incision group: } 22 \\
\text { patients, and endoscopic group: } \\
29 \text { patient) }\end{array}$ & 1 year \\
\hline 8 & $\begin{array}{c}\text { Ching-Jen Wang et al,2004 } \\
\text { (18) }\end{array}$ & Prospective Study & $\begin{array}{l}55 \text { (group } 1 \text { autogenous graft: } 23, \\
\text { group } 2 \text { allogenous: } 32 \text { ) }\end{array}$ & 34 months \\
\hline 9 & $\begin{array}{c}\text { Ching-Jen Wang et al,2004 } \\
\text { (19) }\end{array}$ & Prospective Study & $\begin{array}{c}35 \text { (19 single bundle group and } 16 \\
\text { double-bundle groups) }\end{array}$ & 2 years \\
\hline 10 & Thomas Houe et al,2004 (20) & Prospective Study & 16 & 35 (25-51.5) months \\
\hline 11 & $\begin{array}{c}\text { Young bok Jung, et al,2004 } \\
(21)\end{array}$ & $\begin{array}{l}\text { Retrospective } \\
\text { Study }\end{array}$ & 11 & 52 month \\
\hline 12 & $\begin{array}{c}\text { Jin Hwan Ahn et al,2005 } \\
\text { (22) }\end{array}$ & $\begin{array}{l}\text { Retrospective } \\
\text { Study }\end{array}$ & $\begin{array}{c}36 \text { (18 patients received } \\
\text { autogenous double-loop } \\
\text { hamstring /group I and } 18 \\
\text { Achilles tendon allograft /group } \\
\text { II). }\end{array}$ & 2 years \\
\hline 13 & $\begin{array}{c}\text { Kyoung Ho Yoon et al,2005 } \\
\text { (23) }\end{array}$ & Prospective Study & 26 & $\begin{array}{c}25 \text { months (range, } 12 \text { to } 48 \\
\text { months). }\end{array}$ \\
\hline 14 & $\begin{array}{c}\text { LCDR Jon K. Sekiya et } \\
\text { al,2005 (24) }\end{array}$ & $\begin{array}{l}\text { Retrospective } \\
\text { Study }\end{array}$ & 21 & $\begin{array}{c}\text { Mean } 5.9 \text { years (range, } 2.6 \text { to } \\
11 \text { years), }\end{array}$ \\
\hline 15 & $\begin{array}{c}\text { John D. MacGillivray et } \\
\text { al,2006 (25) }\end{array}$ & $\begin{array}{l}\text { Retrospective } \\
\text { Study }\end{array}$ & $\begin{array}{c}20 \text { (13 traditional endoscopic } \\
\text { transtibial group and } 7 \text { tibial inlay } \\
\text { group). }\end{array}$ & $\begin{array}{l}\text { Mean follow-up of } 5.7 \text { years } \\
\text { (range, } 2 \text { to } 15 \text { years) }\end{array}$ \\
\hline 16 & $\begin{array}{c}\text { Yi-Sheng Chan et al,2006 } \\
\text { (26) }\end{array}$ & Prospective Study & 20 & $\begin{array}{l}40 \text { months (range, } 36 \text { to } 50 \\
\text { months }\end{array}$ \\
\hline 17 & $\begin{array}{c}\text { Raffaele Garofalo et al, } 2006 \\
\text { (27) }\end{array}$ & Case Series & 15 & $\begin{array}{l}\text { mean follow-up of } 3.2 \text { years } \\
\text { (range, } 2 \text { to } 5 \text { years) }\end{array}$ \\
\hline 18 & $\begin{array}{c}\text { Chih-Hwa Chen et al,2006 } \\
\text { (28) }\end{array}$ & Prospective Study & 52 & 4 years \\
\hline 19 & $\begin{array}{c}\text { Jong-Keun Seon et al,2006 } \\
(29)\end{array}$ & $\begin{array}{l}\text { Retrospective } \\
\text { Study }\end{array}$ & $\begin{array}{l}43 \text { ( } 21 \text { The transtibial tunnel- } \\
\text { group /group and } 22 \text { the tibial } \\
\text { inlay group /group B ) }\end{array}$ & 2 years \\
\hline 20 & $\begin{array}{l}\text { Nobuo Adachi et al,2007 } \\
(30)\end{array}$ & Prospective Study & $29(22 / 7)$ & 2 years \\
\hline 21 & $\begin{array}{c}\text { Chin-Hsien Wu et al,2007 } \\
\text { (31) }\end{array}$ & Prospective Study & 22 & 66 months (range, 60-76) \\
\hline 22 & $\begin{array}{c}\text { Jinzhong Zhao et al,2007 } \\
\text { (32) }\end{array}$ & $\begin{array}{l}\text { Retrospective } \\
\text { Study }\end{array}$ & $\begin{array}{l}43 \text { (22 patients 7-strand hamstring } \\
\text { graft (7SHG) group and } 21 \\
\text { patients 4-strand hamstring graft } \\
\text { (4SHG). }\end{array}$ & 2 years \\
\hline
\end{tabular}


$23 \quad$ Bin Li et al, 2008 (33)

\section{W. F. M. Jackson et al,2008 (34) \\ 25 To Wong et al,2008 (35)

$\begin{array}{cc}26 & \text { Jinzhong Zhao et al,2008 } \\ (36)\end{array}$

Retrospective Study
36 (4SHG group $(\mathrm{n}=15)$ and a

LARS group $(n=21))$.

Prospective Study

Prospective Study
26

55 (28 A-M trans-tibia group and 27 A-L trans-tibia group)
2 years

10 years

$48 \S 15.9$ months for A-M and $45.0 \S 13.7$ months for A-L.
(37)

$\begin{array}{ccc}28 & \begin{array}{c}\text { Sung-Jae Kim et al,2009 } \\ (38)\end{array} & \begin{array}{c}\text { Retrospective } \\ \text { Study }\end{array} \\ & & \text { Case Series } \\ \mathbf{3 0} & \begin{array}{c}\text { Baicheng Chen et al, 2009 } \\ (39)\end{array} & \text { Case Series } \\ & \begin{array}{c}\text { Stijn Hermans et al,2009 } \\ (40)\end{array} & \\ & & \text { Retrospective } \\ \text { S1 } & \text { Oog Jin Shon et al,2010 (41) }\end{array}$

Case Series

Randomized

Control Trial group and 21 Lateral Side Augmentation group) 29 (8 Transtibial single bundle group; 11 inlay single-bundle group; 10 inlays double-bundle group)

22

25 (9 with a bone-patellar tendonbone autograft (BPTB), 15 with a semitendinosus gracilis (STG) autograft, and 1 with an Achilles tendon allograft )

30 (14 Single bundles tibial inlay/group A and 16 Double bundles tibial inlay/ group B)
42 (21 Medial Side Augmentation
2 years

2 years

46.4 months in Group T, 36.3 months in Group I1, and 29.4 months in Group I2
2 years

Mean follow-up of 9.1 years (range, 6.5-12.6).

Group A mean 90.5 months and group B mean 64 months

\begin{tabular}{|c|c|c|c|c|}
\hline 32 & $\begin{array}{l}\text { Odd Arve Lien et al,2010 } \\
(42)\end{array}$ & $\begin{array}{l}\text { Retrospective } \\
\text { Study }\end{array}$ & 43 & 48 month (17-109) \\
\hline 33 & $\begin{array}{c}\text { Kyoung Ho Yoon et al,2011 } \\
\text { (43) }\end{array}$ & $\begin{array}{l}\text { Randomized } \\
\text { Control Trial }\end{array}$ & $\begin{array}{l}53 \text { (25 Single bundle group and } \\
28 \text { Double bundle group) }\end{array}$ & 2 years \\
\hline 34 & $\begin{array}{c}\text { Rachad Zayni et al, } 2011 \\
\text { (44) }\end{array}$ & $\begin{array}{l}\text { Retrospective } \\
\text { Study }\end{array}$ & 21 & 29 months (range $12-48$ ) \\
\hline 35 & $\begin{array}{c}\text { Yu-Chuan Lin et al,2013 } \\
\text { (45) }\end{array}$ & $\begin{array}{l}\text { Retrospective } \\
\text { Study }\end{array}$ & $\begin{array}{c}59 \text { (25 Bone-patellar tendon-bone } \\
\text { autograft and } 34 \text { hamstring } \\
\text { autograft ) }\end{array}$ & $\begin{array}{l}51.6 \text { months in pPT group } \\
\text { and } 51.1 \text { months in HT } \\
\text { group }\end{array}$ \\
\hline 36 & Sang Hak Lee et al,2013 (46) & $\begin{array}{l}\text { Retrospective } \\
\text { study }\end{array}$ & $\begin{array}{c}89 \text { (34 Transtibial groups,40 SB } \\
\text { inlay group, and 15 DB inlay } \\
\text { group) }\end{array}$ & 24 month \\
\hline 37 & Bin Li et al, 2014 (47) & $\begin{array}{l}\text { Retrospective } \\
\text { Study }\end{array}$ & $\begin{array}{l}37 \text { (18 Hamstring autograft group } \\
\text { and } 19 \text { Tibialis anterior allograft) }\end{array}$ & 2 years \\
\hline 38 & $\begin{array}{l}\text { Seyed Taghi Norbakhsh et } \\
\text { al,2014 (48) }\end{array}$ & Prospective Study & 52 & 3 years \\
\hline 39 & $\begin{array}{c}\text { Eun-Kyoo Song et al, } 2014 \\
\text { (49) }\end{array}$ & Cohort Study & $\begin{array}{l}66 \text { (transtibial with a hamstring } \\
\text { (36 patients) and tibial inlay with } \\
\text { the patellar tendon ( } 30 \text { patients) }\end{array}$ & $\begin{array}{l}148 \text { months (range, } 98-196 \\
\text { months). }\end{array}$ \\
\hline 40 & Daifeng lu et al,2014 (50) & $\begin{array}{l}\text { Randomized } \\
\text { Control Trial }\end{array}$ & $\begin{array}{c}32 \text { (17 improve tibial inlay and } 15 \\
\text { traditional tibial inlay) }\end{array}$ & 1 year \\
\hline 41 & Xiujiang Sun et al,2015 (51) & $\begin{array}{l}\text { Retrospective } \\
\text { Study }\end{array}$ & $\begin{array}{c}71 \text { (36 Autograft group and } 35 \\
\text { allograft group) }\end{array}$ & $\begin{array}{l}\text { The autograft group was } 3.2 \\
\pm 0.2 \text { years and the allograft } \\
\text { group was } 3.3 \pm 0.6 \text { years }\end{array}$ \\
\hline 42 & Vineet Jain et al,2016 (52) & $\begin{array}{l}\text { Retrospective } \\
\text { Study }\end{array}$ & $\begin{array}{l}40 \text { (18 Double bundle group and } \\
22 \text { Single bundle group ) }\end{array}$ & 24 month \\
\hline 43 & Jia Li et al,2016 (53) & $\begin{array}{l}\text { Randomized } \\
\text { Control Trial }\end{array}$ & $\begin{array}{l}80 \text { (26 patients in the autograft } \\
\text { group, } 27 \text { in the hybrid graft } \\
\text { group, and } 27 \text { in the g-irradiated } \\
\text { allograft group ) }\end{array}$ & 5 years \\
\hline 44 & $\begin{array}{c}\text { Terence Wai-kit Chan et } \\
\text { al,2016 (54) }\end{array}$ & $\begin{array}{l}\text { Retrospective } \\
\text { Study }\end{array}$ & 21 & 50 months (24-60 months) \\
\hline 45 & $\begin{array}{l}\text { Rodrigo Salim et al, } 2017 \\
\text { (55) }\end{array}$ & $\begin{array}{l}\text { Retrospective } \\
\text { Study }\end{array}$ & 21 & 4.4 years $(0.6-11$ years $)$ \\
\hline 46 & Rhatomy et al,2019 (56) & $\begin{array}{l}\text { Retrospective } \\
\text { Study }\end{array}$ & 25 & 2 years \\
\hline 47 & D. Saragaglia et al,2019 (57) & $\begin{array}{l}\text { Retrospective } \\
\text { Study }\end{array}$ & $\begin{array}{l}16 \text { (8 hamstring group;8 LARS } \\
\text { group) }\end{array}$ & 24 month \\
\hline
\end{tabular}


Range of Motion. Seventeen studies (511 patients, 29.8\%) did not describe the range of motion after surgery. Thus, only 30 studies (1079 patients, $63.06 \%$ ) reported a range of motion after surgery.

Knee extension deficit was evaluated using three categories; grade 1: nearly normal $<3^{\circ}$, grade 2: $3-5^{\circ}$, and grade $3:>6^{\circ}$. Among the studies that reported range of motion outcomes, 96 patients $(9.15 \%)$ experienced the loss of extension $\left(<3^{\circ}=59(61.4 \%), 3-5^{\circ}=29(30.2 \%)\right.$, $>6^{\circ}=2(0.2 \%)$, and the degree of the loss of extension was not reported for five patients).

Knee flexion deficit was evaluated using four categories; grade 1 : nearly normal $<5^{\circ}$, grade 2 : 6 $15^{\circ}$, grade $3: 16-25^{\circ}$, and grade 4 (severe flexion deficit) $>25^{\circ}$. Three hundred and twelve patients $(28.9 \%)$ experienced the loss of flexion $\left(<5^{\circ}=134\right.$ $(42.9 \%), 6-15^{\circ}=60(19.2 \%), 16-25^{\circ}=4(1.2 \%)$, severe flexion deficit $\left(>25^{\circ}=8(2.5 \%)\right)$, and the degree of the loss of range of motion was not reported for 106 patients (Table 2).

Table 2. Outcomes Measures of Posterior Cruciate Ligament Reconstruction.

\begin{tabular}{|c|c|c|c|c|c|c|}
\hline No & $\begin{array}{c}\text { Author and } \\
\text { Year }\end{array}$ & Graft Type & $\begin{array}{c}\text { PCLR Technique } \\
\text { and Fixation Device }\end{array}$ & $\begin{array}{c}\text { Sample Size } \\
\text { (male/female) }\end{array}$ & $\begin{array}{l}\text { Range of } \\
\text { Motion } \\
\text { Outcome } \\
\end{array}$ & Knee Laxity Outcome \\
\hline 1 & $\begin{array}{l}\text { P. P. Mariani } \\
\text { et al, } 1997 \\
(11)\end{array}$ & BPTB Autograft & $\begin{array}{l}\text { Single bundle PCL } \\
\text { reconstruction Both } \\
\text { ends of the graft } \\
\text { were secured with } \\
\text { interference screws }\end{array}$ & $24(16 / 8)$ & $\begin{array}{c}18 \text { patients } \\
(75 \%) \text { complete } \\
\text { ROM } 2 \text { patients } \\
(8 \%) \\
\text { experienced a } \\
\text { lack of } \\
\text { extension of } \\
\text { between } 3^{\circ} \text { and } \\
5^{\circ} 6 \text { patients } \\
(25 \%) \text { loss of } \\
\text { flexion, between } \\
6^{\circ} \text { and } 15^{\circ} 4 \text { pts } \\
\text { loss of } \\
\text { extension of less } \\
\text { than } 3^{\circ} \text {. }\end{array}$ & $\begin{array}{l}\text { KT } 2000 \text { Measurement } \\
\text { 0-2mm: 6;3-5 mm: } \\
\text { 13; 6-10: 3;> 10: } 2\end{array}$ \\
\hline 2 & $\begin{array}{l}\text { Sung-Jae } \\
\text { Kim et al, } \\
2000(12)\end{array}$ & $\begin{array}{l}\text { Group 1; BPTB } \\
\text { autograft Group } \\
\text { 2: 11BPTB } \\
\text { allograft and } 34 \\
\text { BPTB autograft }\end{array}$ & $\begin{array}{l}\text { Single Bundle PCL } \\
\text { Reconstruction } \\
\text { Femoral Fixation: } \\
\text { Interference Screw } \\
\text { Tibial Fixation: } \\
\text { Interference Screw }\end{array}$ & $55(42 / 13)$ & $\begin{array}{l}1 \text { patient in } \\
\text { group I and } 10 \\
\text { patients in } \\
\text { group II lost } \\
\text { terminal flexion, } \\
\text { an average of } \\
10^{\circ} \text { (range, } 5^{\circ} \text { to } \\
20^{\circ} \text { ). There was } \\
\text { no extension } \\
\text { loss or } \\
\text { extension lag at } \\
\text { the last follow- } \\
\text { up. }\end{array}$ & $\begin{array}{l}\mathrm{KT}-1000 \text { or KT-2000 } \\
\text { arthrometer (testing at } \\
20 \text {-lb force) was } 2.10 \\
\mathrm{~mm} \text { (range, } 1 \text { to } 4 \mathrm{~mm} \text { ) } \\
\text { in group I and } 2.38 \\
\mathrm{~mm} \text { (range, } 0 \text { to } 6 \mathrm{~mm} \text { ) } \\
\text { in group II }\end{array}$ \\
\hline 3 & $\begin{array}{c}\text { John Nyland } \\
\text { et al,2002 } \\
\text { (13) }\end{array}$ & $\begin{array}{l}\text { allograft } \\
\text { (anterior tibialis } \\
\text { tendon } n=17 \text {, } \\
\text { semitendinosus- } \\
\text { gracilis tendon } \\
n=2 \text { ) }\end{array}$ & $\begin{array}{l}\text { double-bundle PCL } \\
\text { reconstruction (using } \\
\text { allograft tissue) } \\
\text { Biodegradable } \\
\text { interference screws } \\
\text { were used for all } \\
\text { graft fixation } \\
\text { procedures. }\end{array}$ & $19(14 / 5)$ & $\begin{array}{l}\text { All patients had } \\
\text { normal }(n=19) \\
\text { or near normal } \\
(n=1) \text { passive } \\
\text { knee joint } \\
\text { extension }\left(<3^{\circ}\right) \\
\text { and flexion }(0- \\
\left.5^{\circ} \text { deficient }\right) \\
\text { compared to the } \\
\text { opposite knee } \\
\text { joint. }\end{array}$ & $\begin{array}{l}\text { Posterior drawer tests } \\
\text { at } 70^{\circ} \text { knee flexion } \\
\text { revealed all normal } \\
(n=11) \text { or nearly } \\
\text { normal results }(n=8) \\
\text { Knee arthrometry } \\
\text { measurements showed } \\
2.4 \pm 2 \text { mm posterior } \\
\text { displacement. }\end{array}$ \\
\hline 4 & $\begin{array}{l}\text { Chih-Hwa } \\
\text { Chen et } \\
\text { al,2002 (14) }\end{array}$ & $\begin{array}{l}\text { Hamstring } \\
\text { tendon autograft }\end{array}$ & $\begin{array}{l}\text { Single bundle PCL } \\
\text { reconstruction Both } \\
\text { ends of the graft } \\
\text { were secured with } \\
\text { interference screws }\end{array}$ & $27(18 / 9)$ & $\begin{array}{c}\text { Eighty-five } \\
\text { percent (n: 23) } \\
\text { of the patients } \\
\text { had full ROM, a } \\
\text { 3-degree or less } \\
\text { difference A 3- }\end{array}$ & $\begin{array}{l}\text { Posterior drawer and } \\
\text { posterior sag testing } \\
\text { and KT-1000 } \\
\text { examination } \\
\text { demonstrated: } 8 \text { ( } 29 \%) \\
\text { the patients exhibited a }\end{array}$ \\
\hline
\end{tabular}




\begin{tabular}{|c|c|c|c|c|c|c|}
\hline & & & $\begin{array}{c}\text { and screw and } \\
\text { washer }\end{array}$ & & $\begin{array}{l}\text { to the 5-degree } \\
\text { difference in } \\
\text { extension or a } \\
\text { 6- to a 15- } \\
\text { degree deficit in } \\
\text { flexion from the } \\
\text { opposite limb } \\
\text { was recorded } \\
\text { for } 11 \% \text { (n: } 3 \text { ) } \\
\text { of the patients. } \\
\text { An extension } \\
\text { deficit of more } \\
\text { than } 6 \text { degrees } \\
\text { or a flexion } \\
\text { deficit of more } \\
\text { than } 16 \text { degrees } \\
\text { was found in } \\
\text { one patient } \\
\text { (4\%). }\end{array}$ & $\begin{array}{c}\text { 0- to 2-mm total } \\
\text { anteroposterior } \\
\text { translation. } 15 \text { (56\%) } \\
\text { percent revealed a 3- to } \\
\text { 5-mm ligament laxity. } \\
4 \text { patients (15\%) } \\
\text { demonstrated a 6- to } \\
\text { 10-mm laxity. }\end{array}$ \\
\hline 5 & $\begin{array}{l}\text { Chih-Hwa } \\
\text { Chen et } \\
\text { al,2002 (15) }\end{array}$ & $\begin{array}{l}\text { Quadriceps } \\
\text { tendon autograft } \\
\text { and quadruple } \\
\text { hamstring } \\
\text { tendon } \\
\text { autograft. }\end{array}$ & $\begin{array}{l}\text { Single bundle PCL } \\
\text { reconstruction } \\
\text { Femoral: titanium } \\
\text { interference screw } \\
\text { Tibia: Bicortical } \\
\text { screw and washer } \\
\text { and bioscrew }\end{array}$ & $49(32 / 17)$ & $\begin{array}{l}\text { The normal } \\
\text { rating was } \\
\text { recorded for } \\
77 \%(\mathrm{~N}: 17 \text { ) of } \\
\text { the quadriceps } \\
\text { tendon group } \\
\text { and } 85 \%(\mathrm{~N}: \\
\text { 23) of the } \\
\text { hamstring } \\
\text { tendon group. } \\
\text { The nearly } \\
\text { normal rating } \\
\text { was recorded } \\
\text { for } 18 \% \text { (N: } 4 \text { ) } \\
\text { of the } \\
\text { quadriceps } \\
\text { tendon group } \\
\text { and } 11 \%(\mathrm{~N}: 3 \text { ) } \\
\text { of the hamstring } \\
\text { tendon group. } \\
\text { The abnormal } \\
\text { present for 5\% } \\
\text { (N:1) of } \\
\text { quadriceps } \\
\text { tendon patients } \\
\text { and } 4 \% \text { (N: } 1 \text { ) } \\
\text { of hamstring } \\
\text { tendon patients. }\end{array}$ & $\begin{array}{l}\text { Posterior drawer and } \\
\text { posterior sag testing } \\
\text { and KT-1000 } \\
\text { examination showed } \\
\text { that32\% (N: 7) of the } \\
\text { quadriceps tendon } \\
\text { group and 29\% (N: 8) } \\
\text { of the hamstring } \\
\text { tendon group exhibited } \\
\text { a 0- to 2-mm total } \\
\text { anterior-posterior } \\
\text { translation. 56\% } \\
\text { percent (N: 13) of the } \\
\text { patients in the } \\
\text { quadriceps tendon } \\
\text { group and 56\% (N: } 15 \text { ) } \\
\text { in the hamstring } \\
\text { tendon group revealed } \\
\text { a 3- to 5-mm ligament } \\
\text { laxity. Two patients } \\
\text { (9\%) with quadriceps } \\
\text { tendon graft and } 4 \\
\text { patients (15\%) with } \\
\text { hamstring tendon } \\
\text { grafts showed a 6- to } \\
10 \text {-mm laxity. }\end{array}$ \\
\hline 6 & $\begin{array}{l}\text { Ching-Jen } \\
\text { Wang et } \\
\text { al,2003 (16) }\end{array}$ & $\begin{array}{l}\text { Autografts } \\
\text { (patellar bone- } \\
\text { tendon-bone } \\
\text { and quadriceps } \\
\text { tendon) } \\
\text { Allografts } \\
\text { (Achilles tendon } \\
\text { and patellar } \\
\text { bone-tendon- } \\
\text { bone). }\end{array}$ & $\begin{array}{l}\text { Single bundle PCL } \\
\text { reconstruction Both } \\
\text { ends of the graft } \\
\text { were secured with } \\
\text { interference screws }\end{array}$ & $30(22 / 8)$ & - & $\begin{array}{l}\text { Posterior drawer test } 0 \text { : } \\
\begin{array}{c}16(51.6 \%) ; 1: 12 \\
\begin{array}{c}(38.7 \%) ; 2: 3(9.7 \%) \\
3: 0\end{array}\end{array}\end{array}$ \\
\hline 7 & $\begin{array}{l}\text { Yasumitsu } \\
\text { Ohkoshi et } \\
\text { al,2003 (17) }\end{array}$ & $\begin{array}{l}\text { Autogenous } \\
\text { hamstring } \\
\text { tendons, }\end{array}$ & $\begin{array}{l}\text { Single bundle PCL } \\
\text { reconstruction } \\
\text { Femoral side: endo } \\
\text { button. Tibial side: } \\
\text { screw and spiked } \\
\text { washer. }\end{array}$ & $51(33 / 18)$ & - & $\begin{array}{l}\mathrm{KT}-1000 \text {, the manual } \\
\text { maximum was } 3.95 \pm \\
1.96 \mathrm{~mm} \text { in the } 2- \\
\text { incision group and } \\
2.38 \pm 1.42 \mathrm{~mm} \text { in the } \\
\text { endoscopic group }\end{array}$ \\
\hline
\end{tabular}




\begin{tabular}{|c|c|c|c|c|c|c|}
\hline 8 & $\begin{array}{l}\text { Ching-Jen } \\
\text { Wang et } \\
\text { al,2004 (18) }\end{array}$ & $\begin{array}{l}\text { Autogenous } \\
\text { grafts ( } \\
\text { quadriceps } \\
\text { tendon- patellar } \\
\text { bones and } \\
\text { quadruple } \\
\text { hamstrings } \\
\text { Allogenous } \\
\text { grafts (Achilles } \\
\text { tendon and } \\
\text { anterior tibial } \\
\text { tendons 0 }\end{array}$ & $\begin{array}{l}\text { Single Bundle PCL } \\
\text { Reconstruction } \\
\text { Femoral Fixation: } \\
\text { Bioabsorbable Screw } \\
\text { Tibial Fixation: } \\
\text { Bioabsorbable } \\
\text { Screw, titanium } \\
\text { screw }\end{array}$ & $55(41 / 14)$ & $\begin{array}{c}\text { Range of } \\
\text { Motion: } \\
\text { Autograft } \\
\text { group: Mean: } \\
125 \pm 14^{\circ} \\
\text { Range } 80-140^{\circ} \\
\text { Allograft group: } \\
\text { mean: } 127 \pm 6^{\circ} \\
\text { range } 115-135^{\circ}\end{array}$ & $\begin{array}{c}\text { Posterior Drawer: } \\
\text { 1.Autograft group: } \\
0.92 \pm 0.69(0-3) 2 . \\
\text { Allograft group: } 0.61 \pm \\
0.58(0-2) \text { KT-1000 } \\
\text { 1.Autograft group: } \\
3.16 \pm 2.60(1-10) 2 . \\
\text { Allograft group: } 2.83 \pm \\
1.70(1-6)\end{array}$ \\
\hline 9 & $\begin{array}{l}\text { Ching-Jen } \\
\text { Wang et } \\
\text { al,2004 (19) }\end{array}$ & $\begin{array}{l}\text { Hamstring } \\
\text { tendon autograft }\end{array}$ & $\begin{array}{l}\text { Single and double- } \\
\text { bundle posterior } \\
\text { cruciate ligament } \\
\text { (PCL) Both ends of } \\
\text { the graft were } \\
\text { secured with } \\
\text { bioabsorbable } \\
\text { interference }\end{array}$ & $35(26 / 9)$ & $\begin{array}{c}\text { SB: } 126 \pm 12 \\
(90-140) \text { DB: } \\
124 \pm 14(80- \\
140)\end{array}$ & $\begin{array}{c}\text { Posterior drawer: SB: } \\
1.16 \pm 0.6(0-2) ; \mathrm{DB}: \\
1.13 \pm 0.6(0-2) \mathrm{KT} \\
1000: \mathrm{SB}: 7.1 \pm 3.7(3- \\
\text { 15); DB: } 6.7 \pm 4.5(2- \\
16)\end{array}$ \\
\hline 10 & $\begin{array}{c}\text { Thomas } \\
\text { Houe et } \\
\text { al,2004 (20) }\end{array}$ & $\begin{array}{l}\text { BPTB Autograft } \\
\text { Hamstring } \\
\text { tendon autograft }\end{array}$ & $\begin{array}{l}\text { A posterior cruciate } \\
\text { ligament (PCL) with } \\
\text { one versus two } \\
\text { tunnels femoral One } \\
\text { tunnel-group both } \\
\text { ends of the graft } \\
\text { were secured with } \\
\text { interference screws } \\
\text { Two tunnel-group; } \\
\text { femoral side: } \\
\text { endobutton, tibial } \\
\text { side: interference } \\
\text { screw }\end{array}$ & $16(6 / 8)$ & - & $\begin{array}{l}\text { One tunnel group:: } 30 \\
\text { deg: } 2(2-4) ; 70 \text { deg: } 2 \\
\quad(2-4) \text { Two tunnel } \\
\text { group: } 30 \text { deg: } 3(1.3- \\
3.8) ; 70 \text { deg: } 3(1.3-4)\end{array}$ \\
\hline 11 & $\begin{array}{l}\text { Young bok } \\
\text { jung,et } \\
\text { al,2004 (21) }\end{array}$ & BPTB Autograft & $\begin{array}{l}\text { Single bundle PCL } \\
\text { reconstruction } \\
\text { Femoral Fixation: } \\
\text { Interference Screw } \\
\text { Tibial Fixation: } \\
\text { Screw and washer }\end{array}$ & 11 & - & $\begin{array}{c}\text { The mean side-to-side } \\
\text { difference in } \\
\text { displacement (and } \\
\text { standard deviation) } \\
\text { was } 3.4+/-2.4 \mathrm{~mm} \text { on } \\
\text { the stress radiographs } \\
\text { and } 1.8+/-1.2 \mathrm{~mm} \text { as } \\
\text { measured with the KT- } \\
1000 \text { arthrometer. }\end{array}$ \\
\hline 12 & $\begin{array}{c}\text { Jin Hwan } \\
\text { Ahn et } \\
\text { al,2005 (22) }\end{array}$ & $\begin{array}{l}\text { Autogenous } \\
\text { double-loop } \\
\text { hamstring } \\
\text { tendon (group I) } \\
\text { and Achilles } \\
\text { tendon allograft } \\
\text { (group II). }\end{array}$ & $\begin{array}{l}\text { Single bundle PCL } \\
\text { reconstruction Both } \\
\text { ends of the graft } \\
\text { were secured with } \\
\text { button bioabsorbable } \\
\text { interference screws }\end{array}$ & $36(27 / 9$ & $\begin{array}{l}1 \text { patient who } \\
\text { had received } \\
\text { Achilles tendon } \\
\text { allograft (group } \\
\text { II) had knee } \\
\text { joint stiffness } \\
\text { (range of } \\
\text { motion, } 0^{\circ} \text { to } \\
\left.90^{\circ}\right) .\end{array}$ & $\begin{array}{l}\text { Telos stress test The } \\
\text { group I mean was } 2.2 \\
\text { mm (range, } 0 \text { to } 7 \mathrm{~mm} \text {; } \\
\mathrm{SD}, 1.8 \text { ) and the group } \\
\text { II mean was } 2.9 \mathrm{~mm} \\
\text { (range, } 1 \text { to } 7 \mathrm{~mm} \text {; SD, } \\
1.9 \text { ) }\end{array}$ \\
\hline 13 & $\begin{array}{l}\text { Kyoung Ho } \\
\text { Yoon et } \\
\text { al,2005 (23) }\end{array}$ & $\begin{array}{l}\text { Achilles } \\
\text { allograft }\end{array}$ & $\begin{array}{l}\text { Arthroscopic double- } \\
\text { bundle technique } \\
\text { using a split Achilles } \\
\text { allograft AL bundle } \\
\text { is fixed with } 1 \\
\text { bioabsorbable } \\
\text { interference screw } \\
\text { using an outside-in } \\
\text { method. PM bundle } \\
\text { is fixed with } \\
\text { bioabsorbable } \\
\text { interference screw } \\
\text { and outside-in } \\
\text { method. The } 2\end{array}$ & $26(19 / 7)$ & - & $\begin{array}{l}\text { Radiographic Side-to- } \\
\text { Side Differences of } \\
\text { Posterior Tibial } \\
\text { Translation 0-2 mm: } \\
\text { 18;3-5 mm: 6;6-10 } \\
\text { mm: } 3 ;>10 \mathrm{~mm}: 0\end{array}$ \\
\hline
\end{tabular}




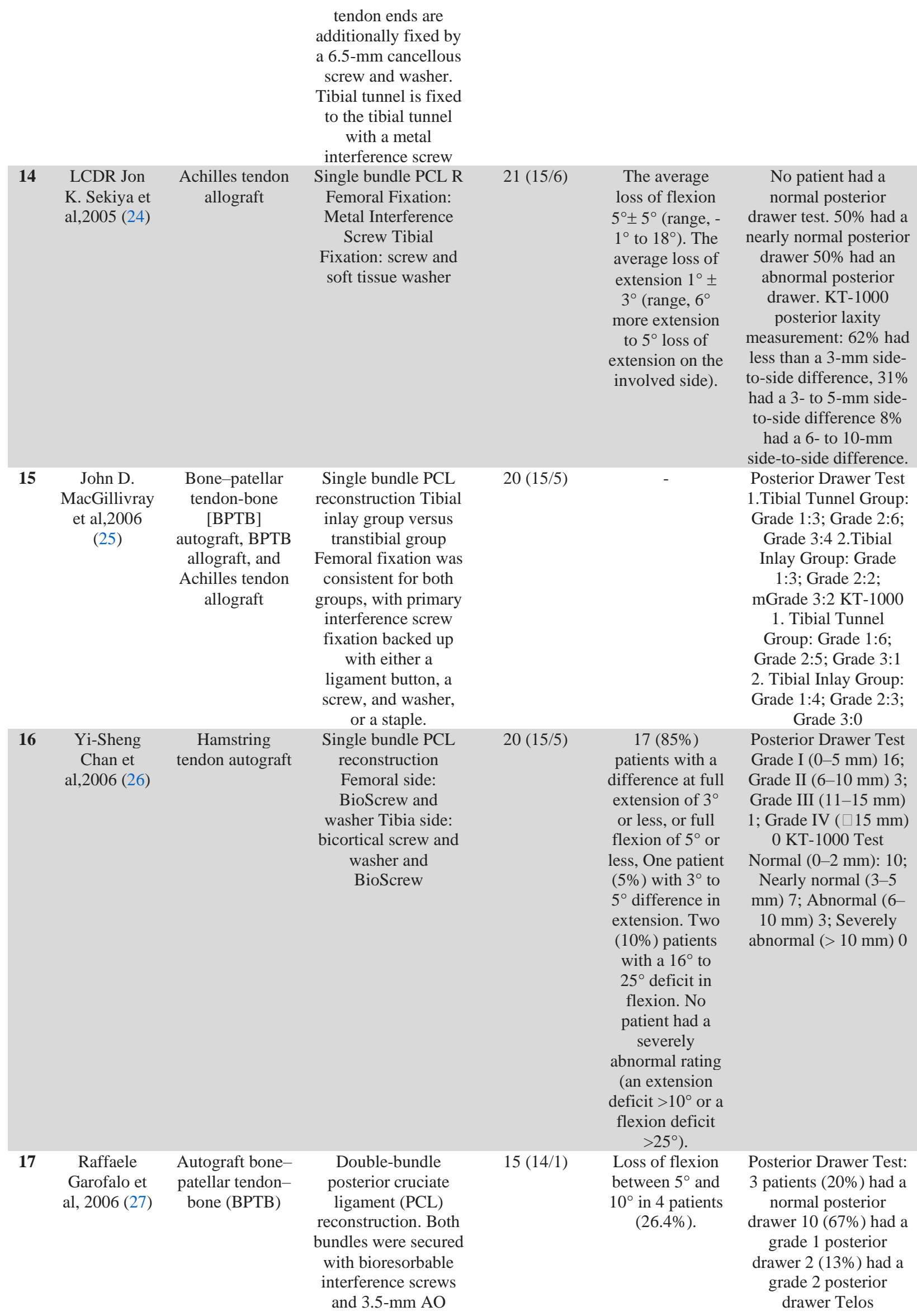


cortical screw with a metallic washer at the tibia.
19

Jong-Keun
Seon et
al,2006 (29)

Quadruple
hamstring
tendon autograft

Quadrupled hamstring autograft, bonepatellar tendonbone autograft
Single bundle PCL reconstruction Both ends of the graft were secured with interference screws and screw and washer
Single bundle PCL reconstruction

Fixation Transtibia group Femoral side using an LA Screw Tibia side: bioabsorbable interference screw Tibial inlay Femoral side: interference screw Tibial side: screw and washer

$52(35 / 17)$

$34(65 \%)$ patients were rated as having normal status. $11(21 \%)$ patients who presented flexion deficit 6 (11\%) patients presented extension deficit were rated as nearly normal. 1 patient $(2 \%)$ had a $16^{\circ}-25^{\circ}$ deficit in flexion.

\section{$43(36 / 7)$}

(lexion.

Radiography: the mean value of posterior translation was 8.06 $\mathrm{mm}$ (range, 5 to 13 $\mathrm{mm}$; SD, $3.7 \mathrm{~mm}$ ) and the mean side-to-side difference was $5.9 \mathrm{~mm}$ (range, 2 to $12 \mathrm{~mm}$; $\mathrm{SD}, 2.63 \mathrm{~mm}$ ).

Posterior drawer test Grade I (0-5 mm): 42; Grade II (6-10 mm): 10; Grade III (11-15 $\mathrm{mm}$ ): 0; Grade IV ([15 $\mathrm{mm}) 0 \mathrm{KT}-1000$ measurement Normal (0-2 mm): 32; Nearly normal (3-5 mm): 10 ; Abnormal (6-10 mm): 8; Severely abnormal (>10 mm): 2

\section{Posterior Drawer \\ Transtibia: Grade I (0- $5 \mathrm{~mm}$ ): 19; Grade II (6-10 mm): 2; Grade III (>10 mm): 0 Tibia inlay: Grade I (0-5 $\mathrm{mm})$ :20; Grade II (6- $10 \mathrm{~mm}$ ): 2; Grade III (>10 mm): 0}

Telos Device (20 N)

Mean side-to-side differences were $3.7 \pm$ 2.1 at the final followup in group $\mathrm{A}$ and 3.3 $\pm 1.6 \mathrm{~mm}$ in group $\mathrm{B}$ Tranastibia: 0-2 mm: 2; 3-5 mm: 14; 6-10 mm: 5 ; >10 mm: 0

Tibial inlay: $0-2 \mathrm{~mm}$ : 3; 3-5 mm:15; 6-10 mm: 4;>10 mm: 0

Stress radiology: mean $3.5 \mathrm{~mm} \pm 2.7$ Posterior laxity measured by

KT-2000 mean 3.7 mm \pm 2.4 ,

KT-1000 examination:

Grade 0 - 2 mm: 10

(46\%) patients, grade 3 to $5 \mathrm{~mm}: 8(36 \%)$ patients, grade $>5$ mm: 4 (18\%) patients. 


\begin{tabular}{|c|c|c|c|c|c|c|}
\hline & & & & & $\begin{array}{c}\text { flexion) in } 2 \\
\text { patients }(9 \%) .1 \\
\text { patient }(4.5 \%) \\
\text { severely } \\
\text { abnormal (a } \\
\text { flexion deficit } \\
\text { of more than } \\
\left.25^{\circ}\right) .\end{array}$ & \\
\hline 22 & $\begin{array}{c}\text { Jinzhong } \\
\text { Zhao et } \\
\text { al,2007 (32) }\end{array}$ & $\begin{array}{l}\text { Hamstring } \\
\text { tendon autograft }\end{array}$ & $\begin{array}{l}\text { Single bundle } \\
\text { posterior cruciate } \\
\text { ligament (PCL) The } \\
\text { femoral side: mini- } \\
\text { plate Tibial side } \\
\text { titanium button or } \\
\text { screw post }\end{array}$ & $43(34 / 9)$ & $\begin{array}{l}\text { Normal } \\
\text { hyperextension } \\
\text { of } 5^{\circ} \text { was lost in } \\
\text { two } 4 \mathrm{SHG} \\
\text { patients and one } \\
7 \mathrm{SHG} \text {, Loss of } \\
5^{\circ} \text { of full } \\
\text { flexion occurred } \\
\text { in two } 4 \mathrm{SHG} \\
\text { patients and one } \\
7 \mathrm{SHG}\end{array}$ & $\begin{array}{c}\text { Posterior Drawer 4HS } \\
\text { Group: Grade 1: 11; } \\
\text { Grade:2 5; Grade 3: 4; } \\
\text { Grade 4:1 7HS Group: } \\
\text { Grade 1: 15; Grade 2: } \\
\text { 5; Grade 3: 2; Grade 4: } \\
\text { 0 KT 1000 } \\
\text { Examination: 4HS } \\
\text { Group: Grade 1: 12; } \\
\text { Grade 2: 5; Grade 3: 4; } \\
\text { Grade 4: } 0 \text { 7HS Group: } \\
\text { Grade 1: } 17 \text {; Grade 2: } \\
\text { 4; Grade 3: 1; Grade 4: } \\
0\end{array}$ \\
\hline 23 & $\begin{array}{l}\text { Bin Li et al, } \\
2008(33)\end{array}$ & $\begin{array}{c}\text { Fur-strand } \\
\text { hamstring graft } \\
\text { autograft and a } \\
\text { LARS artificial } \\
\text { ligament. }\end{array}$ & $\begin{array}{l}\text { Single bundle PCL } \\
\text { reconstruction Both } \\
\text { ends of the graft } \\
\text { were secured with } \\
\text { interference screws } \\
\text { and screw and } \\
\text { washer }\end{array}$ & $36(30 / 6)$ & - & $\begin{array}{l}\text { Posterior Drawer - } \\
\text { 4HS Group: Grade 1: } \\
\text { 6; Grade 2:5; Grade 3: } \\
\text { 4; Grade 4: 0 - LARS } \\
\text { Group: Grade 1: 16; } \\
\text { Grade 2: 5; Grade 3: 0; } \\
\text { Grade 4: } 0 \text { KT 1000 } \\
\text { Examination 4HS } \\
\text { Group: Grade 1: } \\
\text { 4; Grade 2: 3; Grade 3: } \\
\text { 6; Grade 4: } 2 \text { ARS } \\
\text { Group: Grade 1: 10; } \\
\text { Grade 2: 8; Grade 3: 3; } \\
\text { Grade 4: } 0\end{array}$ \\
\hline 24 & $\begin{array}{c}\text { W. F. M. } \\
\text { Jackson et } \\
\text { al,2008 (34) }\end{array}$ & $\begin{array}{l}\text { Hamstring } \\
\text { Tendon } \\
\text { autograft }\end{array}$ & $\begin{array}{l}\text { Single bundle PCL } \\
\text { reconstruction } \\
\text { Femoral fixation: } \\
\text { titanium round-head } \\
\text { cannulated } \\
\text { interference screw } \\
\text { Tibial fixation: RCI } \\
\text { screw }\end{array}$ & $26(25 / 1)$ & $\begin{array}{l}21 \text { patients had } \\
\text { less than } 3^{\circ} \text { of } \\
\text { loss of } \\
\text { extension } 20 \\
\text { patients had less } \\
\text { than } 5^{\circ} \text { of loss } \\
\text { of flexion }\end{array}$ & $\begin{array}{l}8 \text { patients had grade } 0 \\
\text { laxity. } 12 \text { patients had } \\
\text { grade } 1 \text { laxity } 2 \\
\text { patients had grade } 2 \\
\text { laxity. The mean side } \\
\text { to side difference in } \\
\text { posterior translation } \\
\text { was } 1.1 \mathrm{~mm} \text { (SD } 1.9 \text { ) }\end{array}$ \\
\hline 25 & $\begin{array}{l}\text { To Wong et } \\
\text { al,2008 (35) }\end{array}$ & $\begin{array}{c}\text { Hamstring } \\
\text { tendon autograft }\end{array}$ & $\begin{array}{l}\text { Single-bundle PCL } \\
\text { reconstruction Both } \\
\text { bundles were secured } \\
\text { with bioresorbable } \\
\text { interference screws }\end{array}$ & $55(41 / 14)$ & & $\begin{array}{c}\text { Posterior Drawer: } \\
\text { Anteromedial Group: } \\
0.9 \pm 0.5(0-3) \\
\text { Anterolateral group: } \\
0.9 \pm 0.7(0-3) \text { KT- } \\
1000 \text { Examination: } \\
\text { Anteromedial Group: } \\
2.8 \pm 1.6(1-6) \\
\text { Anterolateral group: } \\
3.3 \pm 2.8(1-10)\end{array}$ \\
\hline 26 & $\begin{array}{c}\text { Jinzhong } \\
\text { Zhao et } \\
\text { al,2008 (36) }\end{array}$ & $\begin{array}{c}\text { Autogenous } \\
\text { hamstring } \\
\text { tendons }\end{array}$ & $\begin{array}{l}\text { Single bundle PCL } \\
\text { reconstruction The } \\
\text { femoral tunnel side: } \\
\text { Button Tibia side: } \\
\text { titanium button }\end{array}$ & $18(14 / 4)$ & $\begin{array}{l}1 \text { patient lost the } \\
\text { normal } 5^{\circ} \text { of } \\
\text { hyperextension } \\
2 \text { patients had a } \\
5^{\circ} \text { flexion } \\
\text { limitation. }\end{array}$ & $\begin{array}{c}\text { The side-to-side } \\
\text { difference in posterior } \\
\text { laxity was } 0.7 \pm 0.9 \\
\text { mm. } 17(94.4 \%) \text { had a } \\
\text { negative posterior } \\
\text { drawer test and KT- } \\
1000 \text { examination }\left(90^{\circ}\right. \\
\text { of flexion and } 30 \mathrm{lb}),< \\
3 \mathrm{~mm} .1 \text { patient had a } \\
1+\text { posterior drawer } \\
\text { test and a KT- } 1000\end{array}$ \\
\hline
\end{tabular}




\begin{tabular}{|c|c|c|c|c|c|}
\hline 27 & $\begin{array}{l}\text { Jin-Zhong } \\
\text { Zhao et al, } \\
2009 \text { (37) }\end{array}$ & $\begin{array}{c}\text { Seven strands of } \\
\text { hamstring } \\
\text { tendon } \\
\text { Autograft }\end{array}$ & $\begin{array}{l}\text { Single bundle PCL } \\
\text { reconstruction The } \\
\text { femoral side Button } \\
\text { Tibia side titanium } \\
\text { button }\end{array}$ & $42(33 / 9)$ & $\begin{array}{l}1 \text { patient in each } \\
\text { of the MSA and } \\
\text { LSA group had } \\
5^{\circ} \text { extension } \\
\text { limitation; } 2 \\
\text { patients in each } \\
\text { of the MSA and } \\
\text { LSA groups had } \\
5^{\circ} \text { flexion } \\
\text { limitation }\end{array}$ \\
\hline
\end{tabular}

side-to-side difference of $5 \mathrm{~mm}$.

KT-1000 examination showed MSA group: 0 to $2 \mathrm{~mm}: 15$ patients (78.9\%); 3 to $5 \mathrm{~mm}: 3$

$(15.8 \%)$ and 6 to 10 $\mathrm{mm}: 1(5.3 \%)$, with an average of $1.6 \pm 1.2$ $\mathrm{mm}$. LSA group:0 to 2 $\mathrm{mm}: 14$ patients $(82.3 \%) ; 3$ to $5 \mathrm{~mm}: 2$ $(11.8 \%)$ and 6 to 10 mm: 1 (5.9\%), with an average of $1.5 \pm 1.3$ $\mathrm{mm}$. The posterior drawer test: MSA

Group; Grade 1+: 4 and Grade 2+: 1; LSA Group; Grade 1+: 1; Grade 2+: 2

\begin{tabular}{|c|c|c|c|c|c|c|}
\hline 28 & $\begin{array}{c}\text { Sung-Jae } \\
\text { Kim et } \\
\text { al,2009 (38) }\end{array}$ & $\begin{array}{l}\text { Achilles tendon } \\
\text { allograft }\end{array}$ & $\begin{array}{l}\text { Transtibial single } \\
\text { bundle group (group } \\
\text { T); Arthroscopic } \\
\text { inlay single-bundle } \\
\text { procedure group } \\
\text { (Group I1), } \\
\text { Arthroscopic inlay } \\
\text { double-bundle } \\
\text { procedure (Group I2) } \\
\text { Femoral Fixation: } \\
\text { Bioabsorbable } \\
\text { interference Screw. } \\
\text { Tibial Fixation: } \\
\text { Bioabsorbable } \\
\text { Interference Screw }\end{array}$ & $29(20 / 9)$ & $\begin{array}{c}\text { Final } \\
\text { examination } \\
\text { with a } \\
\text { goniometer } \\
\text { knee flexion to } \\
\text { be } 2.8^{\circ} \pm 0.70^{\circ} \\
\text { in Group } \mathrm{T}, 4.1^{\circ} \\
\pm 2.59^{\circ} \text { in } \\
\text { Group } \mathrm{I} 1 \text {, and } \\
3.4^{\circ} \pm 0.84^{\circ} \text { in } \\
\text { Group } \mathrm{I} 2 \text {. }\end{array}$ & $\begin{array}{c}\text { The mean side-to-side } \\
\text { differences in posterior } \\
\text { tibial translation as } \\
\text { measured with Telos } \\
\text { stress radiography } \\
\text { were } 5.6 \pm 2.00 \mathrm{~mm} \text { in } \\
\text { Group T; } 4.7 \pm 1.62 \mathrm{~mm} \\
\text { in Group I1, and } 3.6 \pm \\
1.43 \mathrm{~mm} \text { in Group I2 }\end{array}$ \\
\hline 29 & $\begin{array}{l}\text { Baicheng } \\
\text { Chen et al, } \\
2009 \text { (39) }\end{array}$ & $\begin{array}{l}\text { Autogenous } \\
\text { hamstring } \\
\text { tendons }\end{array}$ & $\begin{array}{l}\text { Double-bundle } \\
\text { posterior cruciate } \\
\text { ligament (PCL) } \\
\text { reconstruction using } \\
8 \text { strands of } \\
\text { autogenous } \\
\text { hamstring tendon } \\
\text { The grafts were fixed } \\
\text { by the use of a non- } \\
\text { hardware suspension } \\
\text { fixation technique. }\end{array}$ & $22(17 / 5)$ & $\begin{array}{l}1 \text { patient had a } \\
5^{\circ} \text { flexion } \\
\text { limitation, } 1 \\
\text { patient had a } \\
10^{\circ} \text { flexion } \\
\text { limitation } 1 \\
\text { patient who had } \\
\text { a } 5^{\circ} \text { extension } \\
\text { limitation }\end{array}$ & $\begin{array}{l}\text { Posterior Drawer Test. } \\
\text { Grade 0: } 17 \text { patients } \\
(89.5 \%) \text {; Grade } 1+: 1 \\
\text { patient }(5.3 \%) \text {; Grade } \\
2+: 1 \text { patient }(5.3 \%) \\
\text { The mean KT-1000 } \\
\text { examination results } \\
\text { mean } 1.0 \pm 1.0 \mathrm{~mm} \\
\text { postoperatively. The } \\
\text { stress radiography } \\
\text { results in } 2.0 \pm 1.2 \mathrm{~mm} \\
\text { postoperatively }\end{array}$ \\
\hline 30 & $\begin{array}{c}\text { Stijn } \\
\text { Hermans et } \\
\text { al,2009 (40) }\end{array}$ & $\begin{array}{l}\text { Bone-patellar } \\
\text { tendon-bone } \\
\text { autograft } \\
\text { (BPTB), } \\
\text { Semitendinosus } \\
\text { gracilis (STG) } \\
\text { autograft, and } \\
\text { Achilles tendon } \\
\text { allograft }\end{array}$ & $\begin{array}{c}\text { Anterolateral bundle } \\
\text { reconstruction of the } \\
\text { PCL Femoral side: a } \\
\text { cannulated } \\
\text { interference screw } \\
\text { (RCI) Tibial side: } \\
\text { interference screw } \\
\text { (RCI) and a back-up } \\
\text { staple fixation were } \\
\text { used. }\end{array}$ & $25(22 / 3)$ & $\begin{array}{l}\text { A mean loss of } \\
8^{\circ} \text { of flexion in } \\
\text { comparison } \\
\text { with the } \\
\text { contralateral } \\
\text { knee was } \\
\text { present. }\end{array}$ & $\begin{array}{c}\text { The posterior drawer } \\
\text { test results Grade 0: } \\
(\mathrm{n}=2), \text { Grade } \\
\text { 1(n=15),orGrade } \\
\text { 2(n=5) Telos } \\
\text { Radiology BpTB mean } \\
6.2 \text { mm (SD: } 2.6) ; \\
\text { Hamstring mean } 3,9 \\
\text { mm (SD: } 2,6) \text { KT- } \\
\text { 1000 examination. } \\
\text { BpTB: mean } 2.1 \mathrm{~mm} \\
\text { (SD:1.9); Hamstring: } \\
\text { mean } 2,2 \text { mm (SD:1,4) }\end{array}$ \\
\hline 31 & $\begin{array}{c}\text { Oog Jin } \\
\text { Shon et } \\
\text { al,2010(41) }\end{array}$ & $\begin{array}{l}\text { Bone-patellar } \\
\text { tendon-bone } \\
\text { (BPTB) } \\
\text { allografts and }\end{array}$ & $\begin{array}{l}\text { Single bundle tibial } \\
\text { inlay PCL } \\
\text { reconstruction } \\
\text { (Group A) and }\end{array}$ & $30(26 / 4)$ & $\begin{array}{l}1 \text { patient in } \\
\text { group A and } 2 \\
\text { patients in } \\
\text { group B showed }\end{array}$ & $\begin{array}{l}\text { Posterior drawer test } \\
\text { Group A: Grade I (0-5 } \\
\text { mm): 13; Grade II (6- } \\
10 \mathrm{~mm}): 1 \text {; Grade III }\end{array}$ \\
\hline
\end{tabular}


Achilles tendon allografts, and Achilles tendon allografts. double-bundle tibial inlay PCL reconstruction

(Group B) Fixation. Group A. Femoral Side: absorbable interference screw and a staple with an Achilles allograft and a nonabsorbable interference screw with a BPTB

allograft. Tibial side: cancellous screw and washer. Group B:

Femoral Side: absorbable interference screw Tibial side: biointerference screw and a staple

\begin{tabular}{|c|c|c|}
\hline 32 & $\begin{array}{l}\text { Odd Arve } \\
\text { Lien et } \\
\text { al,2010 (42) }\end{array}$ & $\begin{array}{l}\text { Bone-patellar } \\
\text { tendon-bone } \\
\text { (BPTB) } \\
\text { autograft, } \\
\text { allografts and } \\
\text { hamstring } \\
\text { autograft }\end{array}$ \\
\hline 33 & $\begin{array}{l}\text { Kyoung Ho } \\
\text { Yoon et } \\
\text { al,2011 (43) }\end{array}$ & $\begin{array}{l}\text { Achilles } \\
\text { allograft }\end{array}$ \\
\hline
\end{tabular}

$34 \begin{array}{cc}\text { Rachad } \\ \text { Zayni et al, } \\ \text { 2011 (44) }\end{array} \quad \begin{gathered}\text { Quadriceps } \\ \text { tendon autograft }\end{gathered}$

35 $\begin{array}{cc}\text { Yu-Chuan } & \begin{array}{c}\text { Bone-patellar } \\ \text { Lin et }\end{array} \\ \text { tendon-bone }\end{array}$ al,2013 (45) /double bundle PCL

reconstruction Both ends of the graft interference screws

Single bundle PCL reconstruction Femoral Side: cancellous screw and spiked washer or staples, as well as bioabsorbable

interference screws for the double

fixation. Tibial side: metal interference screw (anterolateral bundle)

reconstruction using a quadriceps tendon autograft Femoral side: resorbable interference screw and non-resorbable bicortical screw inserted proximally Tibial side: resorbable interference screw Single Bundle PCL ReconstructionGroup bone-patellar tendonbone graft (PT

Group) and group hamstring graft (HT

Group) Femoral tunnel was fixed first with an interference screw Tibial tunnel was fixed with an
Single bundle were secured with

Single bundle PCL approximately

$10^{\circ}$ of knee

flexion

limitation
(11-15 mm): 0; Grade

IV ([15 mm): 0 Group

B: Grade I (0-5

$\mathrm{mm}): 15$; Grade II (6-

$10 \mathrm{~mm}): 1$; Grade III

(11-15 mm): 0; Grade IV (>15 mm): 0

TELOS Radiography: Group A $3.0 \mathrm{~mm} \pm 1.1$; Group B 2,6 mm \pm 0,49

\begin{tabular}{|c|c|c|}
\hline $43(29 / 14)$ & $\begin{array}{l}\text { Maximum } \\
\text { flexion: } 133 \\
(\mathrm{SD}=7.5)\end{array}$ & $\begin{array}{c}\text { KT } 1000(n: 37): 9.2 \\
\text { mm (SD = 4.1) Stress } \\
\text { Radiograph (n: 41): } 8.4 \\
\text { mm (SD = 4.8) }\end{array}$ \\
\hline $53(45 / 8)$ & $\begin{array}{l}\text { SB Group: } 138^{\circ} \\
63.3^{\circ} \mathrm{DB} \\
\text { Group: } 136^{\circ} 6 \\
4.2^{\circ} \text { Limited } \\
\text { range of motion } \\
\text { was observed as } \\
\text { a postoperative } \\
\text { complication in } \\
1 \text { case of the SB } \\
\text { group ( } 4 \% \text { ) and } \\
2 \text { cases of the } \\
\text { DB group }(7 \%)\end{array}$ & $\begin{array}{c}\text { Telos posterior stress } \\
\text { radiographs SB Group: } \\
4,5 \pm 2,3 \text { DB Group: } \\
3,1 \pm 2,4\end{array}$ \\
\hline $21(18 / 3)$ & $\begin{array}{l}2 \text { patients } \\
(9.5 \%) \\
\text { presented a } \\
\text { moderate } \\
\text { flexion deficit } \\
\text { of } 6^{\circ}-15^{\circ} \text {. }\end{array}$ & $\begin{array}{l}\text { The mean side-to-side } \\
\text { differential posterior } \\
\text { laxity was } 3.6 \mathrm{~mm} \\
\text { (range } 0-7 \text { ) }\end{array}$ \\
\hline
\end{tabular}




\begin{tabular}{|c|c|c|c|c|c|}
\hline & & & $\begin{array}{l}\text { interference screw } \\
\text { (metal interference } \\
\text { was used in PT } \\
\text { group and bio- } \\
\text { absorbable } \\
\text { interference screws } \\
\text { were used in HT } \\
\text { group. The fixation } \\
\text { in each tunnel was } \\
\text { further secured by a } \\
\text { post- screw with a } \\
\text { washer. }\end{array}$ & & $\begin{array}{c}\text { (mm) PT Group: } 2.8 \pm \\
1.6(1-6.5) \text {. HT Group: } \\
2.6 \pm 1.5(1-7.5)\end{array}$ \\
\hline 36 & $\begin{array}{c}\text { Sang Hak } \\
\text { Lee et } \\
\text { al,2013 (46) }\end{array}$ & $\begin{array}{l}\text { Achilles tendon } \\
\text { allograft, } \\
\text { autogenous } \\
\text { hamstring } \\
\text { tendon w }\end{array}$ & $\begin{array}{l}\text { Group 1: ALB } \\
\text { reconstruction using } \\
\text { the transtibial tunnel } \\
\text { technique; Group 2: } \\
\text { ALB reconstruction } \\
\text { using the modified } \\
\text { inlay; and Group 3: } \\
\text { double-bundle } \\
\text { reconstruction using } \\
\text { the modified inlay } \\
\text { technique Fixation: } \\
\text { biodegradable } \\
\text { interference screw at } \\
\text { the femoral tunnel } \\
\text { additionally, and a } \\
\text { post and tie were } \\
\text { made with a screw } \\
\text { and washer in both } \\
\text { the tibial and femoral } \\
\text { sides }\end{array}$ & $89(82 / 7)$ & 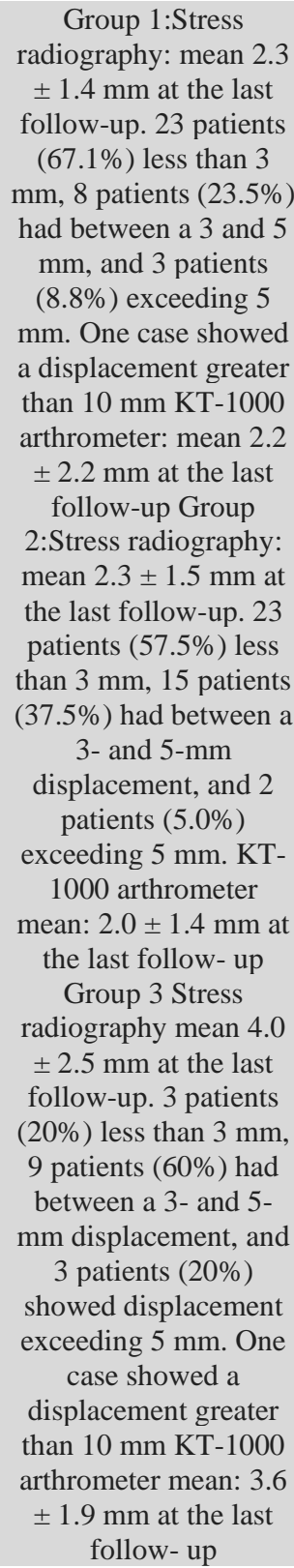 \\
\hline 37 & $\begin{array}{c}\text { Bin Li et al, } \\
2014 \text { (47) }\end{array}$ & $\begin{array}{l}\text { Hamstring } \\
\text { autograft group } \\
\text { and tibialis } \\
\text { anterior } \\
\text { allograft }\end{array}$ & $\begin{array}{l}\text { Single Bundle PCL } \\
\text { Reconstruction } \\
\text { Group A [4-strand } \\
\text { hamstring tendon } \\
\text { autograft (4SHG), } n \\
=18 \text { ] and group B } \\
\text { [2-strand tibialis } \\
\text { anterior allograft }\end{array}$ & $37(25 / 12)$ & $\begin{array}{l}\text { Arthrometer }(\mathrm{mm}) \\
\text { Group A: } 4.1 \pm 1.7 ; \\
\text { Group B: } 3.3 \pm 1.8 \\
\text { Posterior Drawer: } \\
\text { Group A grade } 0: 3 ; \\
\text { grade } 1: 1 ; \text { grade } 2: 4 ; \\
\text { grade } 3: 0 \text { Group B }\end{array}$ \\
\hline
\end{tabular}


(2STAG), $n=19]$

Femoral Fixation: endobutton Tibial

Fixation: cannulated interference screw.

$38 \begin{array}{ccc}\text { Seyed Taghi } & \text { Hamstring } & \text { Single-bundle PCL } \\ \text { Norbakhsh et } & \text { tendon autograft } & \text { reconstruction (the } \\ \text { al,2014 (48) } & & \text { anterolateral part of } \\ & & \text { the PCL) Femoral } \\ & \text { side: Cross pin femur } \\ & \text { Tibial side: bio- } \\ & \text { interference Screw }\end{array}$

grade $0: 8$; grade 1:9; grade 2:2; grade $3: 0$

\section{$52(42 / 10)$ \\ ty-five \\ percent $(n=52)$ full ROM.}

Seven $(13.5 \%)$

of the patients $\mathrm{A}$ 3-5- degree difference in extension or 6 $15^{\circ}$ deficit in flexion. Three patients $(5.7 \%)$ reported severely abnormal knee ROM which was defined as a flexion deficit of more than $25^{\circ}$.

Some patients showed an extension deficit of less than $5^{\circ}$ (5 transtibial cases and 6 tibial inlay cases),

Eun-Kyoo
Song et al,
2014 (49)

2014 (49)

Hamstring and
tibial inlay with
patellar tendon
reconstruction

Transtibial group

Femoral side: the LA screw Tibia side: a bio-interference screw Tibial inlay group Femoral side: interference screw

Tibial side: 2 screws and washers.
$66(58 / 8)$
Laxity: Transtibial group: 30 patients (83.3\%): grade I (0-5 $\mathrm{mm}) ; 6$ patients (16.7\%): grade II (5$10 \mathrm{~mm}$ ) Tibial inlay group: 26 patients (86.7\%): normal or grade I; 4 patients (13.3\%): grade II laxity The mean sideto-side difference (Telos) Transtibial group: $4.1 \mathrm{~mm}$ (range, 0-8 mm) Tibial inlay group: $4.2 \mathrm{~mm}$ (range, 1-8 mm)

\begin{tabular}{|c|c|c|c|c|c|c|}
\hline 40 & $\begin{array}{l}\text { Daifeng lu et } \\
\text { al,2014 (50) }\end{array}$ & $\begin{array}{l}\text { Quadricep } \\
\text { Tendon } \\
\text { autograft }\end{array}$ & $\begin{array}{l}\text { Double bundle PCL } \\
\text { reconstruction (17 } \\
\text { Atroscopic group } \\
\text { and } 15 \text { traditional } \\
\text { tibial inlay group) } \\
\text { Tibial with mini- } \\
\text { steel-plate and tied to } \\
\text { fix the plate onto the } \\
\text { wall of the } \\
\text { anteromedial tibial } \\
\text { Femoral with } \\
\text { interference screw }\end{array}$ & $32(28 / 3)$ & - & $\begin{array}{c}\text { Posterior Drawer test }+ \\
\text { in } 2 \text { pts group } 1 \text { and } 1 \\
\text { pts in group } 2\end{array}$ \\
\hline 41 & $\begin{array}{l}\text { Xiujiang Sun } \\
\text { et al,2015 } \\
(51)\end{array}$ & $\begin{array}{l}\text { Hamstring } \\
\text { tendon autograft } \\
\text { and allograft }\end{array}$ & $\begin{array}{l}\text { Single bundle PCL } \\
\text { reconstruction ( } 36 \\
\text { Autograft group and } \\
35 \text { allograft group) } \\
\text { Both ends of the } \\
\text { graft were secured } \\
\text { with bioabsorbable } \\
\text { interference screws }\end{array}$ & $71(54 / 17)$ & $\begin{array}{c}\text { The average } \\
\text { ROM was } 132.3 \\
\pm 2.2^{\circ} \text { in the } \\
\text { autograft and } \\
134.6 \pm 1.8^{\circ} \text { in } \\
\text { the allograft } \\
\text { group }\end{array}$ & $\begin{array}{c}\text { KT-1000 test } \\
\text { Autograft group: } \\
\text { Grade } 0: 23 \text {; grade } 1: \\
\text { 8; grade } 2: \text { 5; grade } 3: 0 \\
\text { Allograft group: Grade } \\
\text { 0: } 11 \text {; grade } 1: \\
\text { 15; grade } 2: 9 ; \text { grade } 3: \\
0\end{array}$ \\
\hline 42 & $\begin{array}{l}\text { Vineet Jain } \\
\text { et al,2016 } \\
\quad(52)\end{array}$ & $\begin{array}{c}\text { Hamstring } \\
\text { tendon autograft }\end{array}$ & $\begin{array}{l}\text { PCL reconstruction } \\
\text { Single bundle versus } \\
\text { double-bundle PCL } \\
\text { (18 Double bundle }\end{array}$ & $40(40 / 0)$ & - & $\begin{array}{c}\text { KT-1000 (side-to-side } \\
\text { difference in mm) DB } \\
1.78 \mathrm{~mm} \text { (range } 0-6 \\
\text { mm); SB } 2.44 \mathrm{~mm}\end{array}$ \\
\hline
\end{tabular}




\begin{tabular}{|c|c|c|c|c|c|c|}
\hline & & & $\begin{array}{l}\text { group and } 22 \text { Single } \\
\text { bundle group) all } \\
\text { ends of the graft } \\
\text { were secured with } \\
\text { interference screws }\end{array}$ & & & $\begin{array}{c}\text { (range } 0-7 \mathrm{~mm}) \\
\text { Average posterior } \\
\text { translation (kneeling X } \\
\text { ray) DB } 1.33 \mathrm{~mm} \text {; SB } \\
1.95 \mathrm{~mm}\end{array}$ \\
\hline 43 & $\begin{array}{c}\text { Jia Li et } \\
\text { al,2016 (53) }\end{array}$ & $\begin{array}{c}\text { Allograft } \\
\text { (tibialis anterior } \\
\text { tendons) Hybrid } \\
\text { (irradiated } \\
\text { tibialis anterior } \\
\text { tendon allograft } \\
\text { and } \\
\text { semitendinosus } \\
\text { tendon } \\
\text { autograft) } \\
\text { Autograft } \\
\text { (Semitendinosus } \\
\text { and gracilis) }\end{array}$ & $\begin{array}{l}\text { Single bundle PCL } \\
\text { reconstruction } 26 \\
\text { patients in the } \\
\text { autograft group, } 27 \\
\text { in the hybrid graft } \\
\text { group, and } 27 \text { in the } \\
\text { g-irradiated allograft } \\
\text { group Femoral side } \\
\text { EndoButton Tibia } \\
\text { side: bioabsorbable } \\
\text { interference screw }\end{array}$ & $80(50 / 30)$ & - & $\begin{array}{c}\text { Knee Laxity } \\
\text { According to } \\
\text { Instrumented } \\
\text { Anteroposterior } \\
\text { Measurements } \\
\text { Autograft } 2.1 \pm 1.0 \\
\text { Hybrid graft } 2.6 \pm 1.2 \\
\text { g-Irradiated allograft } \\
\text { 3.5 } \pm 1.1 \\
\text { Posterior Drawer Test } \\
\text { Autograft: Grade 0: } 11 \text {; } \\
\text { Grade I: } 15 ; \text { Grade } \\
\text { II:0; Grade III:0 } \\
\text { Hybrid: Grade 0: } 10 ; \\
\text { Grade I: } 16 \text {; Grade } \\
\text { II:1; Grade III:0 } \\
\text { Allograft: Grade 0: 9; } \\
\text { Grade I: } 15 ; \text { Grade } \\
\text { II:3; Grade III:0 }\end{array}$ \\
\hline 44 & $\begin{array}{c}\text { Terence } \\
\text { Wai-kit } \\
\text { Chan et } \\
\text { al,2016 (54) }\end{array}$ & $\begin{array}{l}\text { Quadrupled } \\
\text { hamstrings } \\
\text { autografts }\end{array}$ & $\begin{array}{c}\text { Arthroscopic } \\
\text { transtibial single- } \\
\text { bundle PCL Femoral } \\
\text { side: endo button and } \\
\text { a bioabsorbable } \\
\text { interference screw } \\
\text { Tibial side: screw } \\
\text { post and a } \\
\text { bioabsorbable } \\
\text { interference screw }\end{array}$ & $2121 / 0)$ & - & $\begin{array}{l}\text { Drawer Test: Grade I } \\
(0-5 \mathrm{~mm}): 54.5 \%(12) \text {; } \\
\text { grade II }(5-10 \mathrm{~mm}) \text { : } \\
18.2 \%(4 / 22) .\end{array}$ \\
\hline 45 & $\begin{array}{l}\text { Rodrigo } \\
\text { Salim et al, } \\
2017(55)\end{array}$ & $\begin{array}{l}\text { Autogenous } \\
\text { hamstring } \\
\text { tendons }\end{array}$ & $\begin{array}{l}\text { Single bundle PCL } \\
\text { reconstruction } \\
\text { Femoral side: } \\
\text { interference screw } \\
\text { Tibia side: screw dan } \\
\text { washer }\end{array}$ & 21 & $\begin{array}{c}\text { No deficit of } \\
\text { extension }>4 \\
\text { degrees was } \\
\text { observed in any } \\
\text { patient. The } \\
\text { median range } \\
\text { flexion was } 132 \\
\text { degrees }\end{array}$ & - \\
\hline 46 & $\begin{array}{l}\text { Rhatomy et } \\
\text { al,2019 (56) }\end{array}$ & $\begin{array}{l}\text { Quadrupled } \\
\text { hamstrings } \\
\text { autografts }\end{array}$ & $\begin{array}{c}\text { Single bundle PCL } \\
\text { reconstruction } \\
\text { Femoral side: button } \\
\text { Tibial } \\
\text { side:bioabsorbable } \\
\text { interference screws }\end{array}$ & $25(10 / 15)$ & $\begin{array}{c}3 \text { patients }(21 \%) \\
\text { had ROM } \\
\text { restriction }(0- \\
\left.110^{\circ}\right)\end{array}$ & None \\
\hline 47 & $\begin{array}{c}\text { D. } \\
\text { Saragaglia et } \\
\text { al,2019 (57) }\end{array}$ & $\begin{array}{c}\text { Hamstring } \\
\text { tendon autograft } \\
\text { an artificial } \\
\text { ligament } \\
\text { (ligament } \\
\text { advanced } \\
\text { reinforcement } \\
\text { system } \\
\text { (LARS } ®)\end{array}$ & $\begin{array}{l}\text { Single bundle PCL } \\
\text { reconstruction } 8 \\
\text { using a hamstring } \\
\text { tendon autograft } \\
\text { (hamstring group), } \\
\text { and } 8 \text { using an } \\
\text { artificial ligament } \\
\text { (LARS group) } \\
\text { Femoral side: } \\
\text { interference screw } \\
\text { and two serrated } \\
\text { staples. Tibia side: } \\
\text { absorbable } \\
\text { interference screw }\end{array}$ & $16(15 / 1)$ & - & $\begin{array}{l}\text { X-ray posterior drawer } \\
\text { (mm) hamstring group: } \\
7.37 \mathrm{~mm}(6-8, \mathrm{SD} \\
0.74) \text { and LARS } \\
\text { group: } 5.25 \mathrm{~mm}(3-7, \\
\text { SD } 1.3)\end{array}$ \\
\hline
\end{tabular}

Abbreviation: PBTB: Patellar Bone-Tendon-Bone, SHG/HS: Strand Hamstring Group, MSA: medial side augmentation, LSA: lateral side augmentation, SB: Single Bundle, DB: Double Bundle, ALB: Anterolateral bundle 
Posterior Laxity. In this review, we evaluated laxity using three methods: the posterior drawer displacement test, KT 1000/2000 test, and radiographic stress (Telos) view.

Posterior Drawer Displacement Test. The outcome of the test was grouped into four categories: grade $1(0-5 \mathrm{~mm})$, grade $2(6-10 \mathrm{~mm})$, grade $3(11-15 \mathrm{~mm})$, and grade $4(>15 \mathrm{~mm})$.

Twenty-nine studies involved the posterior drawer displacement test (1051 patients [61.4\%]). According to the posterior drawer test, 682 patients $(64.8 \%)$ still had laxity (grade $1=553$ $(52.6 \%)$, grade $2=109(10.4 \%)$, grade $3=19$ $(1.8 \%)$, and grade $4=1(0.09 \%)$ ).

KT 1000/2000 Arthrometer Test. The outcome of the test was grouped into four categories: grade 1 (normal, $0-2 \mathrm{~mm}$ ), grade 2 (nearly normal, 3-5 mm), grade 3 (abnormal, 6-10 $\mathrm{mm}$ ), and grade 4 (severely abnormal, > $10 \mathrm{~mm}$ ).

Thirty studies reported the posterior drawer displacement in patients (1202 patients, $70.25 \%$ ). The various categories and their corresponding numbers of patients are as follows: grade 1 $($ normal $)=687(57.1 \%)$, grade $2($ nearly normal $)$ $=361(30.0 \%)$, grade 3 (abnormal) $=148$ $(12.3 \%)$, and grade 4 (severely abnormal) $=6$ $(0.49 \%)$. Thus, 515 patients $(42.8 \%)$ still had laxity (grades 2, 3, and 4).

Radiographic Stress (Telos) View. The outcome of the test was grouped into four categories: grade 1 (normal, 0-2 $\mathrm{mm}$ ), grade 2 (nearly normal, 3-5 mm), grade 3 (abnormal, 6-10 $\mathrm{mm}$ ), and grade 4 (severely abnormal, $>10 \mathrm{~mm}$ ).

Eighteen studies reported the radiographic stress (Telos) outcomes in patients (678 patients/ $39.6 \%$ ). The various categories and their corresponding numbers of patients are as follows: grade $1($ normal $)=353(52.06 \%)$, grade 2 (nearly normal $)=228(33.6 \%)$, grade $3($ abnormal $)=95$ $(14.0 \%)$, and grade 4 (severely abnormal) $=2$ $(0.29 \%)$. According to the radiographic stress (Telos) View Measurement, 325 patients (47.9\%) still had laxity (grade 2, 3, and 4).

Comparison of Knee Laxity between the Autograft and Allograft Groups According to the KT 1000/2000 Measurement. Four studies (one randomized controlled trial, two retrospective studies, and one prospective study) reported laxity using the KT 1000/2000 arthrometer measurement. There was no significant difference in outcome between the autograft and allograft groups (MD $=-0.42,95 \%$ CI $[-1.41,0.56], p=0.40$, Figure 2), and there was a high heterogeneity in the groups $\left(\mathrm{I}^{2}=81 \%, \mathrm{p}<\right.$ $0.00001)$. Through the one-by-one elimination of studies, the sensitivity analysis revealed that the heterogeneity remained high.

Comparison of Knee Laxity between Single Bundle and Double Bundle Groups According to the KT 1000/2000 Measurement. Three articles (two prospective studies and one retrospective study) compared the laxity after surgery following the KT 1000/2000 measurement between the single bundle ( $\mathrm{SB} ; \mathrm{n}=$ 49) and double-bundle (DB; $n=42$ ) groups. A random-effects model was applied because of the high statistical heterogeneity $\left(\mathrm{I}^{2}=75 \%, \mathrm{p}=0.02\right)$. There was no significant difference in laxity between the SB and DB groups based on the KT $1000 / 2000$ measurements (MD $=-0.003,95 \% \mathrm{CI}$ $[-1.35,1.29], \mathrm{p}<0.00001$, Figure 3).

According to the Radiographic Stress (Telos) View. Four articles (three prospective studies and one randomized control trial study) compared the laxity after surgery following the radiographic stress (Telos) view between the $S B$ $(n=69)$ and DB $(n=72)$ groups. A fixed-effects model was applied because a low statistical heterogeneity was observed $\left(\mathrm{I}^{2}=34 \%, \mathrm{P}=0.21\right)$. There was a significant difference in laxity outcome between the SB and DB groups based on the radiographic stress (Telos) view ( $\mathrm{MD}=0.69$, $95 \%$ CI $[0.29,1.09], p=0.00008$, Figure 4). This shows that the laxity outcome was significantly higher in the SB group than in the DB group after surgery.

Comparison of Knee Laxity between Transtibial and Tibial Inlay Groups using the Radiographic Stress (Telos) View. Four articles (one prospective study and three retrospective studies) compared laxity after surgery based on the radiographic stress (Telos) view between the Transtibial (TT; $\mathrm{n}=99)$ and Tibial Inlay (TI; $\mathrm{n}=$ 103) groups. A fixed-effects model was applied because a low statistical heterogeneity was observed $\left(\mathrm{I}^{2}=0 \%, \mathrm{p}=0.63\right)$. There was no significant difference in the laxity outcome between the TT and TI groups based on the radiographic stress (Telos) view ( $\mathrm{MD}=0.03$, $95 \%$ CI [-0.33, 0.39], $\mathrm{p}=0.88$, Figure 5). 


\begin{tabular}{|c|c|c|c|c|c|c|c|c|c|c|c|c|}
\hline \multirow[b]{2}{*}{ Study or Subgroup } & \multicolumn{3}{|c|}{ Autograft } & \multicolumn{3}{|c|}{ Allograft } & \multirow{2}{*}{ Weight } & \multirow{2}{*}{$\begin{array}{l}\text { Mean Difference } \\
\text { IV, Random, 95\% CI }\end{array}$} & \multirow{2}{*}{\multicolumn{3}{|c|}{$\begin{array}{c}\text { Mean Difference } \\
\text { IV, Random, } 95 \% \mathrm{Cl}\end{array}$}} & \\
\hline & Mean & SD & Total & Mean & SD & Total & & & & & & \\
\hline Xiujiang Sun 2015 & 3.8 & 1.5 & 36 & 4.8 & 1.7 & 35 & $27.0 \%$ & $-1.00[-1.75,-0.25]$ & & $\longrightarrow$ & & \\
\hline Jia Li 2016 & 2.1 & 1 & 26 & 3.5 & 1.1 & 27 & $28.9 \%$ & $-1.40[-1.97,-0.83]$ & & $\longrightarrow$ & & \\
\hline Ching-Jen Wang2004 & 3.16 & 2.6 & 23 & 2.83 & 1.7 & 32 & $21.5 \%$ & $0.33[-0.88,1.54]$ & & & $-\pi$ & \\
\hline Bin Li 2014 & 4.1 & 1.7 & 18 & 3.3 & 1.8 & 19 & $22.5 \%$ & $0.80[-0.33,1.93]$ & & & & \\
\hline Total $(95 \% \mathrm{CI})$ & & & 103 & & & 113 & $100.0 \%$ & $-0.42[-1.41,0.56]$ & & & & \\
\hline $\begin{array}{l}\text { Heterogeneity: } \mathrm{Tau}^{2}= \\
\text { Test for overall effect: }\end{array}$ & $\begin{array}{l}.79 ; C \\
=0.8\end{array}$ & $\begin{array}{l}i^{2}= \\
(P=\end{array}$ & $\begin{array}{r}15.63 \\
0.40)\end{array}$ & $d f=3$ & (D) & 0.001 ); & $I^{2}=81 \%$ & & -4 & $\begin{array}{l}-2 \\
-2 \\
\text { urs [Allog }\end{array}$ & 0 & 2 \\
\hline
\end{tabular}

Figure 2. Comparison of Knee Laxity between Autograft and Allograft Groups According to KT 1000/2000 measurement

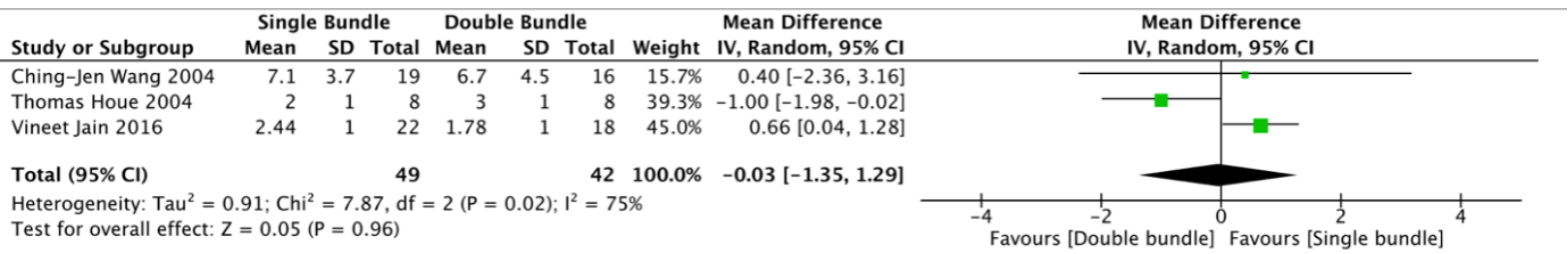

Figure 3. Comparison of Knee Laxity between Single Bundle and Double Bundle Groups According to the KT $1000 / 2000$ measurement

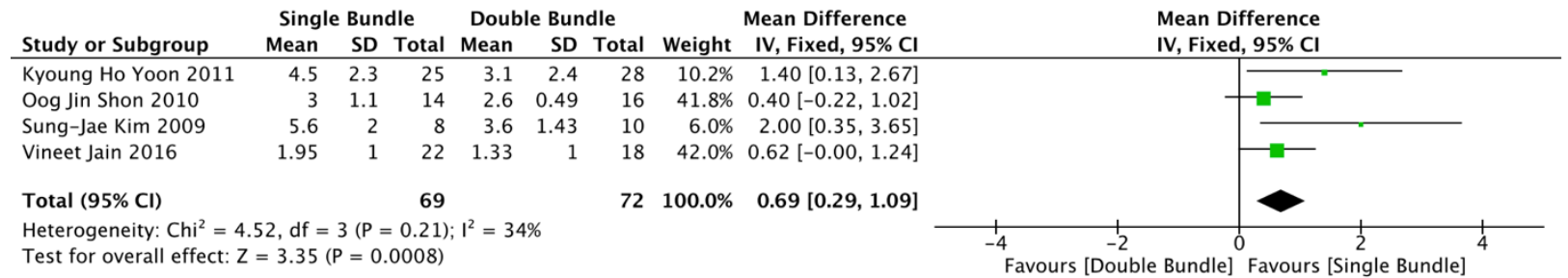

Figure 4. Comparison of Knee Laxity between Single Bundle and Double Bundle Groups According to Radiographic stress (Telos) View

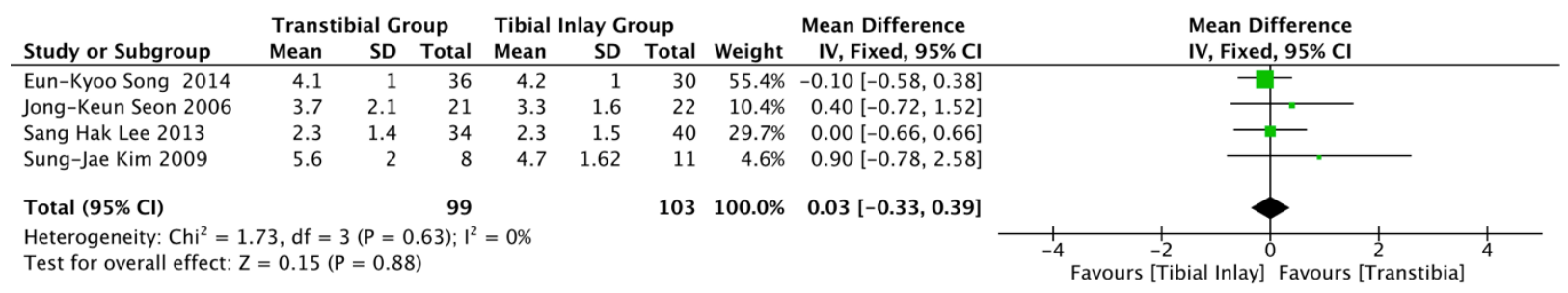

Figure 5. Comparison of Knee Laxity between Transtibial and Tibial Inlay Groups using the Radiographic Stress (Telos) View

\section{DISCUSSION}

The posterior cruciate ligament (PCL) has an important role as a knee joint stabilizer. Some studies have revealed that PCL reconstruction is contributed to enhancing patient knee function. PCL reconstruction aims to restore the normal knee kinematics to improve joint function and to gain nearly normal objective restrain posterior tibial translation post-operatively (40). The indication for surgery in the studies in this review was the failure of conservative treatment or symptomatic PCL rupture with a minimum of 2 positives $(2+)$ posterior drawer test.
The first aim of this review was to evaluate the range of motion after PCL reconstruction. No uniformity was found among studies that evaluated the normal range of motion parameters. There is some agreement to use deficit or loss in knee extension or flexion. In this review, 96 patients $(9.15 \%)$ experienced the loss of extension $\left(<3^{\circ}=59(61.4 \%), 3-5^{\circ}=29(30.2 \%)\right.$, $\left.>6^{\circ}=2(0.2 \%)\right)$, and 312 patients $(28.9 \%)$ experienced the loss of flexion $\left(<5^{\circ}=134\right.$ $(42.9 \%), 6-15^{\circ}=60(19.2 \%), 16-25^{\circ}=4(1.2 \%)$ and severe flexion deficit $\left(>25^{\circ}=8(2.5 \%)\right)$. Some studies stated that knee loss of motion and stiffness post-operative were harder to treat than 
instability. The patient is encouraged to participate in motion exercises and physical therapy to prevent knee loss of function and stiffness (58).

The second aim of this review was to evaluate posterior knee laxity after PCL reconstruction. Posterior knee laxity was evaluated with multiple modalities such as posterior drawer test, stress radiography (Telos) view, and KT-2000 or KT1000 arthrometry. Most studies declared a posterior laxity deterioration after PCL reconstruction.

According to the posterior drawer test outcomes, even though there was a decrease in the grade of laxity after surgery, $64.8 \%$ of the patients still experienced laxity (most patients $(52.6 \%)$ had grade 1 laxity). Some studies $(0.09 \%)$, however, reported severe laxity outcomes (grade 4).

KT 1000/2000 arthrometer measurement. From these studies, we found that $42.8 \%$ of patients still had laxity, and most of them (30\%) had grade 2 (nearly normal) laxity. Radiographic stress (Telos) view showed that $47.9 \%$ of patients still had laxity. Most of them (33.6\%) had grade 2 (nearly normal) laxity.

Young Mo Kim et al. (4) reviewed high-grade isolated PCL rupture that was performed with arthroscopic PCL reconstruction using singlebundle transtibial. This procedure can reduce onegrade posterior knee laxity. Normal or nearly normal knee function was reported by approximately $75 \%$ of patients. Some studies on SB transtibial PCL reconstruction reported improvement in posterior laxity and no stability restoration. MacGillivray et al. reported that whatever method that has been used in tibial fixation (transtibial or inlay) in SB graft PCL reconstruction, could not restore anteroposterior stability of the knee $(25,42)$.

A study by Fanelli et al. revealed that there was 12 of 41 with chronic PCL/ PLC reconstruction that developed abnormality of posterior drawer test in 2 until 10 years (3). Chen et al. concluded that $56 \%$ of patients developed posterior translation of 3 to $5 \mathrm{~mm}$ after PCL reconstruction using $\mathrm{SB}$ quadruple hamstring tendon autograft with a 2-year follow-up (59).

The third goal of this review was to detect the factors that influence laxity or the loss of range of motion after surgery. We performed a sub-group meta-analysis involving the autograft and allograft groups, SB and DB groups, and TT and TI groups.

In this review, the laxity of the knee joint in the autograft and allograft groups was assessed using the KT 1000/ 2000 measurement test. There was no significant difference between the autograft and allograft groups in the outcome $(\mathrm{MD}=-0.42,95 \%$ CI $[-1.41,0.56], \mathrm{p}=0.40$, Figure 2).

A study by Ahn et al revealed that there was a significant radiographic stress view (Telos) in patients who were done PCL reconstruction using SB with either double loop hamstring tendon autograft or Achilles tendon autograft. The postoperative mean displacement was no significant between each group, with $2.2 \mathrm{~mm}$ (range, 0-7 mm) for autograft and $2.9 \mathrm{~mm}$ (range, $1-7 \mathrm{~mm})$ for allograft $(\mathrm{p}=0.14)(34)$. A previous systematic review about the impact of graft origin on joint laxity and activity level post-operative concluded that there was a significant enhancement in functional outcome postoperative, regardless of tendon graft used (2).

We also evaluated the laxity of the knee joint in the SB and DB groups after PCL reconstruction. Three articles provided data on the KT 1000/2000 measurement test. There was no significant difference in the laxity outcome between the SB and DB groups (MD $=-0,003$, $95 \%$ CI $[-1.35,1.29], \mathrm{p}<0.00001$, Figure 3 ). However, four articles reported significant differences in laxity outcomes between the SB and $\mathrm{DB}$ groups $(\mathrm{MD}=0.69,95 \% \mathrm{CI}[0.29,1.09]$, $p=0.00008$, Figure 4) based on the radiographic stress (Telos) view. This shows that the laxity outcome was significantly higher in the SB group than in the DB group after surgery.

A previous systematic review and metaanalysis study by Jorge Chahla et al. reported that PCL procedures using SB or DB has resulted in identical progress in patient-reported outcomes. DB PCL reconstruction was significantly improved in the posterior tibial translation of the knee stability overall based on a randomized controlled clinical trial (60). Another metaanalysis by Dong Yeong Lee et al. revealed that there were no significant differences in side-toside differences between the SB and DB groups (61). According to our review, there is no significant difference in knee stability if measured using the KT 1000/2000; however, the DB technique significantly improved knee stability if measured using Telos radiography. 
Four articles (one prospective study and three retrospective studies) evaluated the laxity of the knee joint based on the radiographic stress (Telos) view in groups that were treated using either the transtibial technique $(n=99)$ or the tibial inlay technique $(\mathrm{n}=103)$. There was no significant difference in the laxity outcome between the Transtibial (TT) and Tibial Inlay (TI) groups $(\mathrm{MD}=0.03,95 \%$ CI $[-0.33,0.39])$.

Similar to the previous systematic review by Young-Soo Shin et al., we did not identify any significant difference in residual laxity between TT and TI technique. All seven enrolled studies compared the Telos radiographs in 149 knees with TT technique and 148 knees with TI techniques. There was no difference in residual posterior laxity between the groups. Knee with grade 2 or greater posterior laxity showed no difference between two groups in the analysis of the five studies (7).

According to our review, there is a loss of extension and flexion deficit after PCL reconstruction $(9.15 \%$ and $28.9 \%$, respectively). Knee laxity was still observed at the final examination based on the results of the posterior drawer test, KT 1000/ 2000 test, and Telos radiographs $(64.8 \%, 42.8 \%$, and $47.9 \%$, respectively). In a subgroup analysis that compared the laxity outcome between groups that were treated using allograft and autograft, SB and $\mathrm{DB}$, and TT and TI, we found no significant differences between groups; however, DB significantly improved knee stability based on Telos radiographic measurements.
Based on the included studies, the keys to successful PCL reconstruction include identifying and treating all pathologies, using strong graft materials, making accurate tunnels placement in the anatomic insertion sites, using a mechanical graft tensioning device, minimizing graft bending, using primary and back-up graft fixation, and using the suitable postoperative rehabilitation protocol.

In conclusion, PCL reconstruction is enhanced with functional outcome scores and joint laxity. Current studies suggest that both the loss of range of motion and laxity still occur after surgery. Further studies are needed to determine the factors that cause the loss of range of motion and laxity and how they can be prevented.

This review has some limitations mainly related to the lack of uniformity. Additionally, few of the included studies emphasize the difficulties encountered when treating this pathology and the need for more high-quality studies.

\section{APPLICABLE REMARKS}

- PCL reconstruction is enhanced with functional outcome scores and joint laxity.

- The loss of range of motion and laxity still occurs after surgery.

- Further studies are needed to determine the factors that cause the loss of range of motion and laxity and how they can be prevented.

\section{ACKNOWLEDGMENT}

We thank Vita Widyasari, Riky Setyawan, and Faiz Alam Rasyid for their help during the manuscript preparation.

\section{REFERENCES}

1. Belk JW, Kraeutler MJ, Purcell JM, McCarty EC. Autograft Versus Allograft for Posterior Cruciate Ligament Reconstruction: An Updated Systematic Review and Meta-analysis. Am J Sports Med. 2018;46(7):1752-1757. doi: 10.1177/0363546517713164 pmid: 28636429

2. Ansari AS, Dennis BB, Horner NS, Zhu M, Brookes C, Khan M, et al. Influence of Graft Source on Postoperative Activity and Joint Laxity in Posterior Cruciate Ligament Reconstruction: A Systematic Review. Arthroscopy. 2019;35(1):262-274 e266. doi: 10.1016/j.arthro.2018.07.027 pmid: 30297155

3. Hudgens JL, Gillette BP, Krych AJ, Stuart MJ, May JH, Levy BA. Allograft versus autograft in posterior cruciate ligament reconstruction: an evidence-based systematic review. J Knee Surg. 2013;26(2):109115. doi: $10.1055 / \mathrm{s}-0032-1319778$ pmid: 23288765

4. Kim YM, Lee CA, Matava MJ. Clinical results of arthroscopic single-bundle transtibial posterior cruciate ligament reconstruction: a systematic review. Am J Sports Med. 2011;39(2):425-434. doi: 10.1177/0363546510374452 pmid: 20702860

5. Kohen RB, Sekiya JK. Single-bundle versus double-bundle posterior cruciate ligament reconstruction. Arthroscopy. 2009;25(12):1470-1477. doi: 10.1016/j.arthro.2008.11.006 pmid: 19962075

6. Panchal HB, Sekiya JK. Open tibial inlay versus arthroscopic transtibial posterior cruciate ligament reconstructions. Arthroscopy. 2011;27(9):1289-1295. doi: 10.1016/j.arthro.2011.04.007 pmid: 21831568 
7. Shin YS, Kim HJ, Lee DH. No Clinically Important Difference in Knee Scores or Instability Between Transtibial and Inlay Techniques for PCL Reconstruction: A Systematic Review. Clin Orthop Relat Res. 2017;475(4):1239-1248. doi: 10.1007/s11999-016-5176-6 pmid: 27896678

8. Tian P, Hu WQ, Li ZJ, Sun XL, Ma XL. Comparison of autograft and allograft tendons in posterior cruciate ligament reconstruction: A meta-analysis. Medicine (Baltimore). 2017;96(27):e7434. doi: 10.1097/MD.0000000000007434 pmid: 28682908

9. Moher D, Liberati A, Tetzlaff J, Altman DG, Group P. Preferred reporting items for systematic reviews and meta-analyses: the PRISMA statement. PLoS Med. 2009;6(7):e1000097. doi: 10.1371/journal.pmed.1000097 pmid: 19621072

10.Longo UG, Rizzello G, Loppini M, Locher J, Buchmann S, Maffulli N, et al. Multidirectional Instability of the Shoulder: A Systematic Review. Arthroscopy. 2015;31(12):2431-2443. doi: 10.1016/j.arthro.2015.06.006 pmid: 26208802

11. Mariani PP, Adriani E, Santori N, Maresca G. Arthroscopic posterior cruciate ligament reconstruction with bone-tendon-bone patellar graft. Knee Surg Sports Traumatol Arthrosc. 1997;5(4):239-244. doi: 10.1007/s001670050057 pmid: 9430574

12. Kim SJ, Shin SJ, Kim HK, Jahng JS, Kim HS. Comparison of 1- and 2-incision posterior cruciate ligament reconstructions. Arthroscop. 2000;16(3):268-278. doi: 10.1016/s0749-8063(00)90051-3

13. Nyland J, Hester P, Caborn DN. Double-bundle posterior cruciate ligament reconstruction with allograft tissue: 2-year postoperative outcomes. Knee Surg Sports Traumatol Arthrosc. 2002;10(5):274-279. doi: 10.1007/s00167-002-0300-4 pmid: 12355300

14. Chen $\mathrm{CH}$, Chen WJ, Shih $\mathrm{CH}$. Arthroscopic reconstruction of the posterior cruciate ligament with quadruple hamstring tendon graft: a double fixation method. J Trauma. 2002;52(5):938-945. doi: 10.1097/00005373-200205000-00020 pmid: 11988663

15. Chen $\mathrm{CH}$, Chen WJ, Shih $\mathrm{CH}$. Arthroscopic reconstruction of the posterior cruciate ligament: a comparison of quadriceps tendon autograft and quadruple hamstring tendon graft. Arthroscopy. 2002;18(6):603-612. doi: 10.1053/jars.2002.32208 pmid: 12098121

16. Wang C, Chen H, Huang T. Outcome of arthroscopic single bundle reconstruction for complete posterior cruciate ligament tear. Inj Int J Care Inj. 2003;34:747-751. doi: 10.1016/S0020-1383(02)00197-3

17. Ohkoshi Y, Nagasaki S, Yamamoto K, Shibata N, Ryosuke Ishida PT, Hashimoto T, et al. Description of a New Endoscopic Posterior Cruciate Ligament Reconstruction and Comparison With a 2-Incision Technique. Arthroscop. 2003;19:825-832. doi: 10.1016/S0749-8063(03)00733-3

18. Wang CJ, Chan YS, Weng LH, Yuan LJ, Chen HS. Comparison of autogenous and allogenous posterior cruciate ligament reconstructions of the knee. Injury. 2004;35(12):1279-1285. doi: 10.1016/j.injury.2003.12.017 pmid: 15561118

19. Wang CJ, Weng LH, Hsu CC, Chan YS. Arthroscopic single- versus double-bundle posterior cruciate ligament reconstructions using hamstring autograft. Injury. 2004;35(12):1293-1299. doi: 10.1016/j.injury.2003.10.033 pmid: 15561120

20.Houe T, Jorgensen U. Arthroscopic posterior cruciate ligament reconstruction: one- vs. two-tunnel technique. Scand J Med Sci Sports. 2004;14(2):107-111. doi: 10.1111/j.1600-0838.2003.00318.x pmid: 15043632

21.Jung YB, Tae SK, Jung HJ, Lee KH. Replacement of the torn posterior cruciate ligament with a midthird patellar tendon graft with use of a modified tibial inlay method. J Bone Joint Surg Am. 2004;86(9). doi: 10.2106/00004623-200409000-00004 pmid: 15342748

22. Ahn JH, Yoo JC, Wang JH. Posterior cruciate ligament reconstruction: double-loop hamstring tendon autograft versus Achilles tendon allograft--clinical results of a minimum 2-year follow-up. Arthroscopy. 2005;21(8):965-969. doi: 10.1016/j.arthro.2005.05.004 pmid: 16084294

23. Yoon KH, Bae DK, Song SJ, Lim CT. Arthroscopic double-bundle augmentation of posterior cruciate ligament using split Achilles allograft. Arthroscopy. 2005;21(12):1436-1442. doi: 10.1016/j.arthro.2005.09.002 pmid: 16376231

24. Sekiya JK, West RV, Ong BC, Irrgang JJ, Fu FH, Harner CD. Clinical outcomes after isolated arthroscopic single-bundle posterior cruciate ligament reconstruction. Arthroscopy. 2005;21(9):10421050. doi: 10.1016/j.arthro.2005.05.023 pmid: 16171628 
25. MacGillivray JD, Stein BE, Park M, Allen AA, Wickiewicz TL, Warren RF. Comparison of tibial inlay versus transtibial techniques for isolated posterior cruciate ligament reconstruction: minimum 2-year follow-up. Arthroscopy. 2006;22(3):320-328. doi: 10.1016/j.arthro.2005.08.057 pmid: 16517317

26. Chan YS, Yang SC, Chang CH, Chen AC, Yuan LJ, Hsu KY, et al. Arthroscopic reconstruction of the posterior cruciate ligament with use of a quadruple hamstring tendon graft with 3- to 5-year follow-up. Arthroscopy. 2006;22(7):762-770. doi: 10.1016/j.arthro.2006.03.020 pmid: 16843813

27. Garofalo R, Jolles BM, Moretti B, Siegrist O. Double-bundle transtibial posterior cruciate ligament reconstruction with a tendon-patellar bone-semitendinosus tendon autograft: clinical results with a minimum of 2 years' follow-up. Arthroscopy. 2006;22(12):1331-1338 e1331. doi: 10.1016/j.arthro.2006.08.003 pmid: 17157733

28. Chen $\mathrm{CH}$, Chuang TY, Wang KC, Chen WJ, Shih CH. Arthroscopic posterior cruciate ligament reconstruction with hamstring tendon autograft: results with a minimum 4-year follow-up. Knee Surg Sports Traumatol Arthrosc. 2006;14(11):1045-1054. doi: 10.1007/s00167-006-0113-y pmid: 16816985

29. Seon JK, Song EK. Reconstruction of isolated posterior cruciate ligament injuries: a clinical comparison of the transtibial and tibial inlay techniques. Arthroscopy. 2006;22(1):27-32. doi: 10.1016/j.arthro.2005.08.038 pmid: 16399457

30. Adachi N, Ochi M, Uchio Y, Iwasa J, Ishikawa M, Shinomiya R. Temporal change of joint position sense after posterior cruciate ligament reconstruction using multi-stranded hamstring tendons. Knee Surg Sports Traumatol Arthrosc. 2007;15(1):2-8. doi: 10.1007/s00167-006-0127-5 pmid: 16799825

31. Wu CH, Chen AC, Yuan LJ, Chang CH, Chan YS, Hsu KY, et al. Arthroscopic reconstruction of the posterior cruciate ligament by using a quadriceps tendon autograft: a minimum 5-year follow-up. Arthroscopy. 2007;23(4):420-427. doi: 10.1016/j.arthro.2006.12.011 pmid: 17418336

32.Zhao J, Huangfu X. Arthroscopic single-bundle posterior cruciate ligament reconstruction: Retrospective review of 4- versus 7-strand hamstring tendon graft. Knee. 2007;14(4):301-305. doi: 10.1016/j.knee.2007.03.008 pmid: 17482468

33.Li B, Wen Y, Wu H, Qian Q, Wu Y, Lin X. Arthroscopic single-bundle posterior cruciate ligament reconstruction: retrospective review of hamstring tendon graft versus LARS artificial ligament. Int Orthop. 2009;33(4):991-996. doi: 10.1007/s00264-008-0628-6 pmid: 18654776

34.Jackson WF, van der Tempel WM, Salmon LJ, Williams HA, Pinczewski LA. Endoscopically-assisted single-bundle posterior cruciate ligament reconstruction: results at minimum ten-year follow-up. $J$ Bone Joint Surg Br. 2008;90(10):1328-1333. doi: 10.1302/0301-620X.90B10.20517 pmid: 18827243

35. Wong T, Wang CJ, Weng LH, Hsu SL, Chou WY, Chen JM, et al. Functional outcomes of arthroscopic posterior cruciate ligament reconstruction: comparison of anteromedial and anterolateral trans-tibia approach. Arch Orthop Trauma Surg. 2009;129(3):315-321. doi: 10.1007/s00402-008-0787-3 pmid: 19034466

36.Zhao J, Xiaoqiao H, He Y, Yang X, Liu C, Lu Z. Sandwich-style posterior cruciate ligament reconstruction. Arthroscopy. 2008;24(6):650-659. doi: 10.1016/j.arthro.2008.01.005 pmid: 18514108

37.Zhao JZ, Huang-Fu XQ, He YH, Yang XG. Single-bundle posterior cruciate ligament reconstruction with remnant preservation: lateral versus medial-sided augmentation technique. Orthop Surg. 2009;1(1):66-73. doi: 10.1111/j.1757-7861.2008.00012.x pmid: 22009784

38. Kim SJ, Kim TE, Jo SB, Kung YP. Comparison of the clinical results of three posterior cruciate ligament reconstruction techniques. J Bone Joint Surg Am. 2009;91(11):2543-2549. doi: 10.2106/JBJS.H.01819 pmid: 19884425

39. Chen B, Gao S. Double-bundle posterior cruciate ligament reconstruction using a non-hardware suspension fixation technique and 8 strands of autogenous hamstring tendons. Arthroscopy. 2009;25(7):777-782. doi: 10.1016/j.arthro.2009.01.017 pmid: 19560642

40. Hermans S, Corten K, Bellemans J. Long-term results of isolated anterolateral bundle reconstructions of the posterior cruciate ligament: a 6- to 12-year follow-up study. Am J Sports Med. 2009;37(8):14991507. doi: $10.1177 / 0363546509333479$ pmid: 19451096

41. Shon OJ, Lee DC, Park CH, Kim WH, Jung KA. A comparison of arthroscopically assisted single and double bundle tibial inlay reconstruction for isolated posterior cruciate ligament injury. Clin Orthop Surg. 2010;2(2):76-84. doi: 10.4055/cios.2010.2.2.76 pmid: 20514264 
42.Lien OA, Aas EJ, Johansen S, Ludvigsen TC, Figved W, Engebretsen L. Clinical outcome after reconstruction for isolated posterior cruciate ligament injury. Knee Surg Sports Traumatol Arthrosc. 2010;18(11):1568-1572. doi: 10.1007/s00167-010-1176-3 pmid: 20571763

43. Yoon KH, Bae DK, Song SJ, Cho HJ, Lee JH. A prospective randomized study comparing arthroscopic single-bundle and double-bundle posterior cruciate ligament reconstructions preserving remnant fibers. Am J Sports Med. 2011;39(3):474-480. doi: 10.1177/0363546510382206 pmid: 21098819

44.Zayni R, Hager JP, Archbold P, Fournier Y, Quelard B, Chambat P, et al. Activity level recovery after arthroscopic PCL reconstruction: a series of 21 patients with a mean follow-up of 29 months. Knee. 2011;18(6):392-395. doi: 10.1016/j.knee.2010.11.005 pmid: 21144755

45.Lin YC, Chen SK, Liu TH, Cheng YM, Chou PP. Arthroscopic transtibial single-bundle posterior cruciate ligament reconstruction using patellar tendon graft compared with hamstring tendon graft. Arch Orthop Trauma Surg. 2013;133(4):523-530. doi: 10.1007/s00402-013-1679-8 pmid: 23344423

46.Lee SH, Jung YB, Lee HJ, Jung HJ, Kim SH. Remnant preservation is helpful to obtain good clinical results in posterior cruciate ligament reconstruction: comparison of clinical results of three techniques. Clin Orthop Surg. 2013;5(4):278-286. doi: 10.4055/cios.2013.5.4.278 pmid: 24340147

47.Li B, Wang JS, He M, Wang GB, Shen P, Bai LH. Comparison of hamstring tendon autograft and tibialis anterior allograft in arthroscopic transtibial single-bundle posterior cruciate ligament reconstruction. Knee Surg Sports Traumatol Arthrosc. 2015;23(10):3077-3084. doi: 10.1007/s00167-014-3267-z pmid: 25193568

48. Norbakhsh ST, Zafarani Z, Najafi A, Aslani H. Arthroscopic posterior cruciate ligament reconstruction by using hamstring tendon autograft and transosseous screw fixation: minimal 3 years follow-up. Arch Orthop Trauma Surg. 2014;134(12):1723-1730. doi: 10.1007/s00402-014-2082-9 pmid: 25186074

49. Seon JK, Song EK, Park H. Comparison of Transtibial and Tibial Inlay Techniques for Posterior Cruciate Ligament Reconstruction with an Average of 10 Year- Follow-up. Orthopaed J Sport Med. 2014;2(2):2325967114S2325900071. doi: 10.1177/2325967114S00071

50.Lu D, Xiao M, Lian Y, Zhou Y, Liu X. Comparison of the operation of arthroscopic tibial inlay and traditional tibial inlay for posterior cruciate ligament reconstruction. Int J Clinic Experiment Med. 2014;7(10):3193-3201.

51. Sun X, Zhang J, Qu X, Zheng Y. Arthroscopic posterior cruciate ligament reconstruction with allograft versus autograft. Arch Med Sci. 2015;11(2):395-401. doi: 10.5114/aoms.2015.50971 pmid: 25995757

52. Jain V, Goyal A, Mohindra M, Kumar R, Joshi D, Chaudhary D. A comparative analysis of arthroscopic double-bundle versus single-bundle posterior cruciate ligament reconstruction using hamstring tendon autograft. Arch Orthop Trauma Surg. 2016;136(11):1555-1561. doi: 10.1007/s00402-016-2512-y pmid: 27438377

53.Li J, Kong F, Gao X, Shen Y, Gao S. Prospective Randomized Comparison of Knee Stability and Proprioception for Posterior Cruciate Ligament Reconstruction With Autograft, Hybrid Graft, and gamma-Irradiated Allograft. Arthroscopy. 2016;32(12):2548-2555. doi: 10.1016/j.arthro.2016.04.024 pmid: 27282110

54. Chan TW, Kong CC, Del Buono A, Maffulli N. Acute augmentation for interstitial insufficiency of the posterior cruciate ligament. A two to five year clinical and radiographic study. Muscles Ligaments Tendons J. 2016;6(1):58-63. doi: 10.11138/mltj/2016.6.1.058 pmid: 27331032

55. Salim R, Nascimento FMD, Ferreira AM, Oliveira LFL, Fogagnolo F, Kfuri M. Tibial Onlay Posterior Cruciate Ligament Reconstruction: Surgical Technique and Results. J Knee Surg. 2018;31(3):284-290. doi: 10.1055/s-0037-1603336 pmid: 28582784

56. Rhatomy S, Saspraditya E, Setyawan R. Arthroscopic Standard Anterior and Posteromedial Portal Posterior Cruciate Ligament Reconstruction With Remnant Preservation: 2-Year Follow-up. Open Sports Sci. 2019;12:44-49. doi: 10.2174/1875399X01912010044

57. Saragaglia D, Francony F, Gaillot J, Pailhe R, Rubens-Duval B, Lateur G. Posterior cruciate ligament reconstruction for chronic lesions: clinical experience with hamstring versus ligament advanced reinforcement system as graft. Int Orthop. 2020;44(1):179-185. doi: 10.1007/s00264-019-04434-7 pmid: 31673741 
58. Cooper DE, Stewart D. Posterior cruciate ligament reconstruction using single-bundle patella tendon graft with tibial inlay fixation: 2- to 10-year follow-up. Am J Sports Med. 2004;32(2):346-360. doi: 10.1177/0363546503261511 pmid: 14977658

59.Fanelli GC, Beck JD, Edson CJ. Arthroscopic double-bundle posterior cruciate ligament reconstruction surgical technique. J Knee Surg. 2010;23(2):89-94. doi: 10.1055/s-0030-1267467 pmid: 21141685

60. Chahla J, Moatshe G, Cinque ME. Single-Bundle and Double-Bundle Posterior Cruciate Ligament Reconstructions: A Systematic Review and Meta-analysis of 441 Patients at a Minimum 2 Years' Followup. Arthroscop. 2017;33(11):2066-2080. doi: 10.1016/j.arthro.2017.06.049

61.Lee DY, Park YJ. Single-Bundle versus Double-Bundle Posterior Cruciate Ligament Reconstruction: A Meta-Analysis of Randomized Controlled Trials. Knee Surg Relat Res. 2017;29(4):246-255. doi: 10.5792/ksrr. 17.050 pmid: 29172385 